\title{
Direct C-Arylation of Free (NH)-Indoles and Pyrroles Catalyzed by Ar-Rh(III) Complexes Assembled In Situ
}

\author{
Xiang Wang, Benjamin S. Lane, Dalibor Sames* \\ Department of Chemistry, Columbia University, 3000 Broadway, New York, New York, 10027
}

\section{Supporting Information}

Materials and General Methods. Small-scale $(<100 \mathrm{mg})$ arylation reactions were carried out in capped glass vials (VWR, $8 \mathrm{~mL}$ ) equipped with a Teflon-coated septum, heated in a 34-well reaction block (Chemglass). Large-scale ( $>1 \mathrm{~g})$ arylation reactions were performed in resalable pressure tubes equipped with a Teflon cap and glass plunger (Ace glass), heated in an oil bath. NMR spectra were obtained on a Bruker spectrometer operating at $400 \mathrm{MHz}$ or $300 \mathrm{MHz}$ for ${ }^{1} \mathrm{H} \mathrm{NMR}, 100 \mathrm{MHz}$ or $75 \mathrm{MHz}$ for ${ }^{13} \mathrm{C}\left\{{ }^{1} \mathrm{H}\right\} \mathrm{NMR}$ and $121 \mathrm{MHz}$ for ${ }^{31} \mathrm{P} \mathrm{NMR}$ in $\mathrm{CDCl}_{3}$ unless otherwise noted. IR spectra were obtained on a Bomen MB-100 FT IR spectrometer. Gas chromatography was performed on an Agilent Technologies $6890 \mathrm{~N}$ gas chromatograph equipped with a $25 \mathrm{~m}$ polydimethylsiloxane capillary column and 5973 Mass Selective Detector. Flash column chromatography was performed employing 200-400 mesh silica gel (EM). Thin layer chromatography (TLC) was performed on silica gel $60 \mathrm{~F}_{254}$ eluting with a 5:1 mixture of hexanes and ethyl acetate unless noted otherwise. $\left[4-\left(\mathrm{CF}_{3}\right) \mathrm{C}_{6} \mathrm{H}_{4}\right]_{3} \mathrm{P} \quad(\mathbf{L})^{1}$ and $\left[\mathrm{Rh}(\mathrm{coe})_{2} \mathrm{Cl}\right]_{2}(\mathbf{1})^{2}$ were synthesized employing known procedures and were as effective as those obtained commercially from Strem. CsOPiv, ${ }^{3} 1$-indole- $d_{l},{ }^{4} 2$-indole- $d_{l}{ }^{5}$ and 3indole- $d_{1}^{5}$ were synthesized employing known procedures. 1,4-dioxane (Anhydrous, Aldrich), haloarenes (Aldrich) and all other reagents were obtained commercially and used as received. 
Table 1. Complete Substrate and Functional Group Scope. ${ }^{a}$

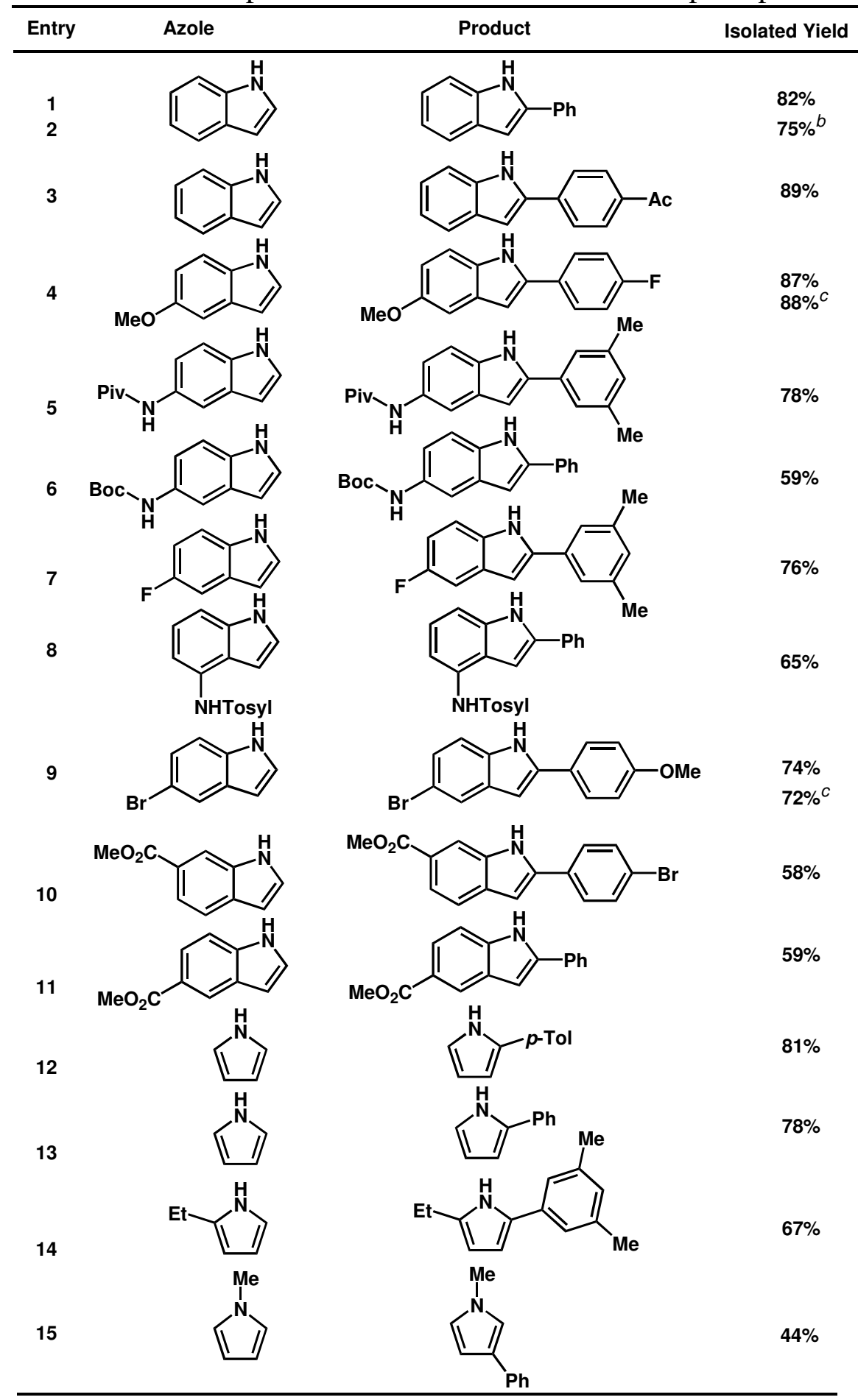

${ }^{a}$ Reaction conditions: 1 equiv of azole, ArI (1.2 equiv), CsOPiv (1.4 equiv), 1 (2.5 mol\%), L (15 mol\%). Dioxane $/ 120{ }^{\circ} \mathrm{C} / 18-36 \mathrm{~h}, \mathrm{~L}=\left[p-\left(\mathrm{CF}_{3}\right)-\mathrm{C}_{6} \mathrm{H}_{4}\right]_{3} \mathrm{P} .{ }^{b} 1.17 \mathrm{~g}$ indole, 1 (1 mol\%), $\mathrm{L}(6 \mathrm{~mol} \%)$, all reagents weighed in air, $120{ }^{\circ} \mathrm{C} / 56 \mathrm{~h} .{ }^{c}$ All reagents weighed in air. 


\section{Synthesis of Functionalized Indole Substrates}

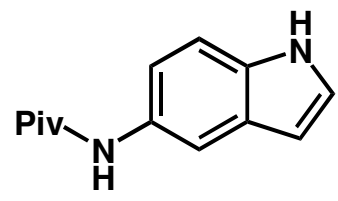

$N$-(5-Indolyl)-2,2-dimethyl-propionamide: Trimethyl acetyl chloride (300 mg, 2.29 mmol) was added to the mixture of 5-aminoindole (264 mg, $2.00 \mathrm{mmol}$ ), triethylamine (1 $\mathrm{mL})$ and $\mathrm{CH}_{2} \mathrm{Cl}_{2}(5 \mathrm{~mL})$. The resulting suspension was stirred at room temperature for 3 $\mathrm{h}$, quenched by $\mathrm{H}_{2} \mathrm{O}(10 \mathrm{~mL})$, extracted (EtOAc, $\left.10 \mathrm{~mL} \times 3\right)$, dried $\left(\mathrm{MgSO}_{4}\right)$ and concentrated. Ether $(15 \mathrm{~mL})$ was then added to precipitate colorless needles, which were further washed by ether $(10 \mathrm{~mL} \times 3)$ to give $N$-(5-indolyl)-2,2-dimethyl-propionamide (298 mg, 69\%) as white needles. ${ }^{1} \mathrm{H}$ NMR (300 MHz, DMSO-d $)$ : $\delta 10.98(\mathrm{~s}, 1 \mathrm{H}), 9.02$ $(\mathrm{s}, 1 \mathrm{H}), 7.79(\mathrm{~s}, 1 \mathrm{H}), 7.20-7.30(\mathrm{~m}, 3 \mathrm{H}), 6.36(\mathrm{~s}, 1 \mathrm{H}), 1.24(\mathrm{~s}, 9 \mathrm{H}) .{ }^{13} \mathrm{C} \mathrm{NMR}(75$ MHz, DMSO- $\left.d_{6}\right): \delta$ 175.4, 132.4, 130.7, 127.0, 125.4, 116.1, 112.1, 110.5, 100.8, 27.5. LRMS (APCI) for $\mathrm{C}_{13} \mathrm{H}_{17} \mathrm{~N}_{2} \mathrm{O}\left(\mathrm{MH}^{+}\right)$calcd (found): 217.13 (217.23).

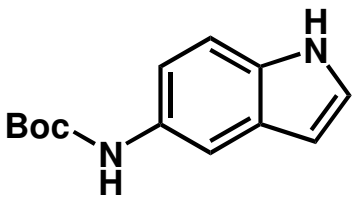

$N$-(5-Indolyl)-carbamic acid tert-butyl ester: A solution of 5-aminoindole (264 mg, $2.00 \mathrm{mmol})$ and di(t-butyl)oxide dicarbonyl $(450 \mathrm{mg}, 2.06 \mathrm{mmol})$ in EtOAc (15 mL) was stirred at room temperature for $12 \mathrm{~h}$, diluted (EtOAc, $30 \mathrm{~mL})$, washed $\left(\mathrm{H}_{2} \mathrm{O}, 10 \mathrm{~mL} \times 3\right)$, dried $\left(\mathrm{MgSO}_{4}\right)$ and concentrated. The resulting oil was dry loaded to a column, and flash chromatographed (2:1 Hexanes:EtOAc) to give $N$-(5-indolyl)-carbamic acid tert-butyl ester (441 mg, 95\%) as a white solid. ${ }^{1} \mathrm{H}$ NMR (300 MHz, $\left.\mathrm{CDCl}_{3}\right): \delta 8.47$ (broad s, 1 H), 7.76 (broad s, 1 H), 7.16-7.27 (m, 3 H), 6.66 (broad s, 1 H), 6.53 (broad s, 1 H), 1.67 $(\mathrm{s}, 9 \mathrm{H}) .{ }^{13} \mathrm{C} \mathrm{NMR}\left(100 \mathrm{MHz}, \mathrm{CDCl}_{3}\right): \delta$ 133.2, 120.9, 128.4, 125.6, 116.2, 112.0, 111.7, 102.7, 80.6, 29.2. IR (film, $\mathrm{cm}^{-1}$ ): 3320, 2978, 1706, 1540, 1483, 1327, 1249, 1166, 1052, 886, 761, 719. LRMS (APCI) for $\mathrm{C}_{13} \mathrm{H}_{17} \mathrm{~N}_{2} \mathrm{O}_{2}\left(\mathrm{MH}^{+}\right)$calcd (found): 233.13 (233.25). 


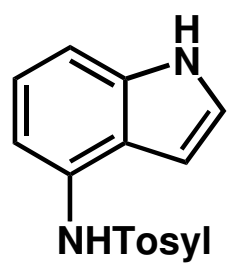

$N$-(4-Indolyl)-4-methyl-benzenesulfonamide: A suspension of tosyl chloride (200 $\mathrm{mg}, 1.10 \mathrm{mmol})$ in $\mathrm{CH}_{2} \mathrm{Cl}_{2}(1 \mathrm{~mL})$ was added slowly to the suspension of 5-aminoindole (132 mg, $1.00 \mathrm{mmol})$ in $\mathrm{CH}_{2} \mathrm{Cl}_{2}(1 \mathrm{~mL})$ at $0{ }^{\circ} \mathrm{C}$. The resulting mixture was warmed up to room temperature, stirred for 30 minutes, quenched $\left(\mathrm{H}_{2} \mathrm{O}, 15 \mathrm{~mL}\right.$ ), extracted (EtOAc, 15 $\mathrm{mL} \times 3)$, dried $\left(\mathrm{MgSO}_{4}\right)$, concentrated and chromatographed (3:1 $\rightarrow 2: 1$ hexanes:EtOAc) to give $\mathrm{N}$-(4-indolyl)-4-methyl-benzenesulfonamide (156 mg, 43\%) as a tan solid. IR (film, $\left.\mathrm{cm}^{-1}\right): 3403,3267,1700,1503,1156,751 .{ }^{1} \mathrm{H}$ NMR $\left(300 \mathrm{MHz}\right.$, methanol- $\left.d_{4}\right): \delta 7.66(\mathrm{~d}$, $\mathrm{J}=8.1 \mathrm{~Hz}, 2 \mathrm{H}), 7.24(\mathrm{~d}, \mathrm{~J}=8.1 \mathrm{~Hz}, 2 \mathrm{H}), 7.16(\mathrm{~d}, \mathrm{~J}=3.6 \mathrm{~Hz}, 1 \mathrm{H}), 7.04(\mathrm{t}, \mathrm{J}=7.8 \mathrm{~Hz}, 1$ H), $6.96(\mathrm{~d}, \mathrm{~J}=7.5 \mathrm{~Hz}, 1 \mathrm{H}), 6.46(\mathrm{~d}, \mathrm{~J}=3.0 \mathrm{~Hz}, 1 \mathrm{H}), 2.39$ (s, $3 \mathrm{H}) .{ }^{13} \mathrm{C} \mathrm{NMR}(75 \mathrm{MHz}$, methanol- $\left.d_{4}\right): \delta$ 143.6, 137.7, 137.6, 129.3, 129.0, 127.3, 124.3, 123.6, 121.3, 114.0, 109.1, 99.0. LRMS (APCI) for $\mathrm{C}_{15} \mathrm{H}_{15} \mathrm{~N}_{2} \mathrm{O}_{2} \mathrm{~S}\left(\mathrm{MH}^{+}\right)$calcd (found): 287.09 (287.25). 


\section{Arylation of Indoles and Pyrroles: Representative Procedures}

Small scale: In an argon-filled glovebox, indole (29.3 mg, $0.25 \mathrm{mmol}), 1$ (4.5 mg, $6.3 \mu \mathrm{mol})$, [4-( $\left.\left(\mathrm{CF}_{3}\right) \mathrm{C}_{6} \mathrm{H}_{4}\right]_{3} \mathrm{P}(\mathbf{L}, 18.0 \mathrm{mg}, 39 \mu \mathrm{mol})$, CsOPiv (82.0 mg, $\left.0.35 \mathrm{mmol}\right)$ and $\mathrm{PhI}(61.0 \mathrm{mg}, 0.30 \mathrm{mmol})$ were charged into a vial, followed by the addition of 1,4dioxane $(0.2 \mathrm{~mL})$. The vial was capped, brought out the glovebox, and heated at $120{ }^{\circ} \mathrm{C}$ in a reaction block for $24 \mathrm{~h}$. The reaction mixture was cooled to room temperature, and passed through a short plug of silica gel $(3 \mathrm{~cm} \times 0.5 \mathrm{~cm}, \mathrm{~L} \times \mathrm{ID})$, eluting with EtOAc (10 $\mathrm{mL}$ ). The resulting solution was concentrated, dry loaded to a column, and flash chromatographed (15:1, hexanes:EtOAc) to give 2-phenyl indole $(39.6 \mathrm{mg}, 82 \%)$ as a white powder.

Small scale (Reagents weighed in air): On benchtop, 1 (4.5 mg, $6.3 \mu \mathrm{mol})$, [4$\left.\left(\mathrm{CF}_{3}\right) \mathrm{C}_{6} \mathrm{H}_{4}\right]_{3} \mathrm{P}(\mathbf{L}, 18.0 \mathrm{mg}, 39 \mu \mathrm{mol})$, CsOPiv (82.0 mg, $\left.0.35 \mathrm{mmol}\right)$, 5-bromoindole (49.0 mg, $0.25 \mathrm{mmol})$ and 4-iodoanisole $(70.2 \mathrm{mg}, 0.30 \mathrm{mmol})$ were rapidly charged into a vial. The vial was subsequently capped, evacuated with a needle through the septum, refilled with argon, and 1,4-dioxane $(0.2 \mathrm{~mL})$ was then added via a syringe through the septum. The vial was then heated at $120{ }^{\circ} \mathrm{C}$ in a reaction block for $24 \mathrm{~h}$. The reaction mixture was cooled to room temperature, and passed through a short plug of silica gel $(3 \mathrm{~cm} \times 0.5 \mathrm{~cm}, \mathrm{~L} \times \mathrm{ID})$, eluting with EtOAc $(10 \mathrm{~mL})$. The resulting solution was concentrated, dry loaded to a column, and flash chromatographed $(8: 1 \rightarrow 5: 1$, hexanes:EtOAc) to give 5-bromo-2-(4-methoxyphenyl) indole (53.5 mg, 72\%) as a pale yellow solid.

Gram scale: On bench top, a mixture of indole (1.17g, $10.0 \mathrm{mmol}), 1(71.7 \mathrm{mg}, 0.10$ mmol), [4- $\left.\left(\mathrm{CF}_{3}\right) \mathrm{C}_{6} \mathrm{H}_{4}\right]_{3} \mathrm{P}(279 \mathrm{mg}, 0.30 \mathrm{mmol})$, CsOPiv (3.26 g, $\left.14.0 \mathrm{mmol}\right)$ and $\mathrm{PhI}$ $(2.45 \mathrm{~g}, 12.0 \mathrm{mmol})$ were charged into into a $20 \mathrm{~mL}$ threaded pressure tube (Ace glass). The tube was then capped, evacuated, refilled with argon, and 1,4-dioxane $(8 \mathrm{~mL})$ was added under a flow of argon. The tube was purged with argon for 2 min, capped and heated at $120{ }^{\circ} \mathrm{C}$ with vigorous stirring. After $52 \mathrm{~h}$, the reaction mixture were cooled to room temperature and filtered through a plug of silica gel $(4 \mathrm{~cm} \times 5 \mathrm{~cm}, \mathrm{~L} \times \mathrm{ID})$, eluting with EtOAc $(30 \mathrm{~mL})$. The resulting solution was concentrated to give a dark colored solid. 
This solid was then washed with hexanes/ether $(50: 1,10 \mathrm{~mL} \times 3)$ afforded 2-phenyl indole $(1.45 \mathrm{~g}, 75 \%)$ as a tan powder ( $>98 \%$ pure by GC-MS).

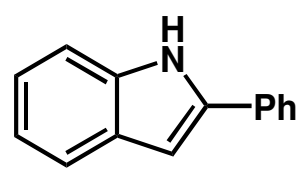

2-Phenyl indole: ${ }^{6}{ }^{1} \mathrm{H}$ NMR (300 MHz, DMSO- $\left.d_{6}\right): \delta 11.6(\mathrm{~s}, 1 \mathrm{H}), 7.87(\mathrm{~d}, J=8.4 \mathrm{~Hz}$, $2 \mathrm{H}), 7.52$ (d, $J=7.8 \mathrm{~Hz}, 1 \mathrm{H}), 7.41-7.49$ (m, $3 \mathrm{H}), 7.29-7.34$ (m, $1 \mathrm{H}), 7.09-7.14$ (m, 1 H), 6.99-7.04 (m, $1 \mathrm{H}), 6.91(\mathrm{~d}, J=0.9 \mathrm{~Hz}, 1 \mathrm{H}) .{ }^{13} \mathrm{C}$ NMR $\left(75 \mathrm{MHz}\right.$, DMSO- $\left.d_{6}\right): \delta$ 131.3, 136.8, 131.9, 128.3, 127.0, 124.7, 121.2, 119.7, 110.1, 111.0, 98.4. IR (film, $\mathrm{cm}^{-1}$ ): 3435, 1451, 741, 689. LRMS (APCI) for $\mathrm{C}_{14} \mathrm{H}_{12} \mathrm{~N}\left(\mathrm{MH}^{+}\right)$calcd (found): 194.10 (194.21).

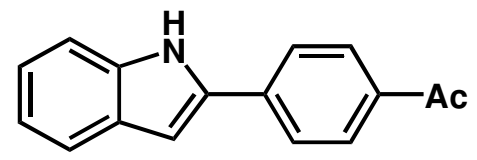

2-(4-Acetylphenyl)-indole: ${ }^{6}{ }^{1} \mathrm{H}$ NMR $\left(300 \mathrm{MHz}\right.$, acetone- $\left.d_{6}\right)$ : $\delta 10.91(\mathrm{~s}, 1 \mathrm{H}), 8.05-$ $8.16(\mathrm{~m}, 4 \mathrm{H}), 7.69$ (d, $J=5.1 \mathrm{~Hz}, 1 \mathrm{H}), 7.52$ (d, $J=5.1 \mathrm{~Hz}, 1 \mathrm{H}), 7.24(\mathrm{t}, J=6.6 \mathrm{~Hz}, 1$ $\mathrm{H}), 7.17-7.26(\mathrm{~m}, 2 \mathrm{H}), 2.70(\mathrm{~s}, 3 \mathrm{H}) .{ }^{13} \mathrm{C}$ NMR $\left(75 \mathrm{MHz}\right.$, acetone- $\left.d_{6}\right): \delta$ 196.7, 138.3, $137.2,137.0,136.2,129.5,129.4,125.2,123.0,121.0,120.3,111.8,101.5,26.2$. IR (film, $\mathrm{cm}^{-1}$ ): 3351, 1669, 1602, 1427, 1192, 791. LRMS (APCI) for $\mathrm{C}_{16} \mathrm{H}_{14} \mathrm{NO}\left(\mathrm{MH}^{+}\right)$ calcd (found): 236.11 (236.26).

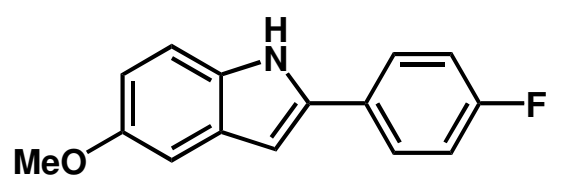

2-(4-Fluoro-phenyl)-5-methoxy-indole: ${ }^{1} \mathrm{H}$ NMR (300 MHz, DMSO- $\left.d_{6}\right)$ : $\delta 11.39$ (s, 1 H), $7.86(\mathrm{dd}, J=2.7,8.7 \mathrm{~Hz}, 2 \mathrm{H}), 7.26-7.32(\mathrm{~m}, 3 \mathrm{H}), 7.01(\mathrm{~d}, J=1.5 \mathrm{~Hz}, 1 \mathrm{H}), 6.73-$ $6.77(\mathrm{~m}, 2 \mathrm{H}), 3.75(\mathrm{~s}, 3 \mathrm{H}) .{ }^{13} \mathrm{C}$ NMR $\left(75 \mathrm{MHz}, \mathrm{DMSO}-d_{6}\right): \delta \quad 162.6,159.4,153.2$, $136.8,131.9,128.7,126.5\left(\mathrm{~d}, J_{\mathrm{F}-\mathrm{C}}=7.8 \mathrm{~Hz}\right), 115.5\left(\mathrm{~d}, J_{\mathrm{F}-\mathrm{C}}=21.4 \mathrm{~Hz}\right), 111.6\left(\mathrm{~d}, J_{\mathrm{F}-\mathrm{C}}=\right.$ 8.7 Hz), 101.3, 98.4, 55.2. IR (film, $\mathrm{cm}^{-1}$ ): 3432, 1589, 1425, 1213, 792. LRMS (APCI) for $\mathrm{C}_{15} \mathrm{H}_{13} \mathrm{~N}\left(\mathrm{MH}^{+}\right)$calcd (found): 242.10 (242.25). 


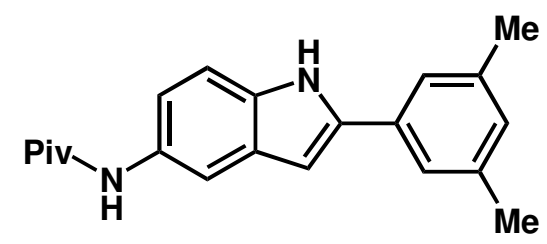

$N$-[2-(3,5-dimethyl-phenyl)-1H-5-indolyl]-2,2-dimethyl-propionamide: ${ }^{1} \mathrm{H}$ NMR (300 MHz, DMSO- $\left.d_{6}\right): \quad \delta 11.31(\mathrm{~s}, 1 \mathrm{H}), 8.99(\mathrm{~s}, 1 \mathrm{H}), 7.76(\mathrm{~s}, 1 \mathrm{H}), 7.45(\mathrm{~s}, 2 \mathrm{H}), 7.23-7.25$ $(\mathrm{m}, 2 \mathrm{H}), 6.92$ (s, $1 \mathrm{H}), 6.78$ (s, $1 \mathrm{H}), 2.33$ (s, $3 \mathrm{H}), 1.24$ (s, $9 \mathrm{H}) .{ }^{13} \mathrm{C}$ NMR $(75 \mathrm{MHz}$, DMSO- $\left.d_{6}\right): \delta$ 176.8, 139.1, 138.7, 134.8, 132.9, 132.3, 129.7, 129.2, 123.6, 117.7, 112.9, 111.5, 99.4, 28.3, 21.9. IR (film, $\mathrm{cm}^{-1}$ ): 3331, 2993, 1702, 1508, 1150. LRMS (APCI) for $\mathrm{C}_{21} \mathrm{H}_{25} \mathrm{~N}_{2} \mathrm{O}\left(\mathrm{MH}^{+}\right)$calcd (found): 321.20 (321.37).<smiles>CC(C)(C)OC(=O)Nc1ccc2[nH]c(-c3ccccc3)cc2c1</smiles>

(2-Phenyl-5-indolyl)-carbamic acid tert-butyl ester: ${ }^{1} \mathrm{H}$ NMR $\left(300 \mathrm{MHz}, \mathrm{CDCl}_{3}\right)$ : $\delta$ 8.29 (s, $1 \mathrm{H}), 7.63-7.67$ (m, $3 \mathrm{H}), 7.43$ (t, $J=7.5 \mathrm{~Hz}, 3 \mathrm{H}), 7.26-7.34$ (m, $2 \mathrm{H}), 7.09$ (dd, $J$ $=1.8,8.7 \mathrm{~Hz}, 1 \mathrm{H}), 6.74(\mathrm{~d}, J=1.5 \mathrm{~Hz}, 1 \mathrm{H}), 6.46(\mathrm{~s}, 1 \mathrm{H}) .{ }^{13} \mathrm{C} \mathrm{NMR}\left(100 \mathrm{MHz}, \mathrm{CDCl}_{3}\right)$ : $\delta$ 154.9, 139.0, 132.6, 131.6, 129.9, 129.3, 128.0, 125.5, 116.3, 111.4, 100.4, 80.5, 29.1. IR (film, $\mathrm{cm}^{-1}$ ): 3321, 2972, 1698, 1478, 1159, 760. LRMS (APCI) for $\mathrm{C}_{19} \mathrm{H}_{21} \mathrm{NO}_{2}\left(\mathrm{MH}^{+}\right)$ calcd (found): 309.16 (309.35).

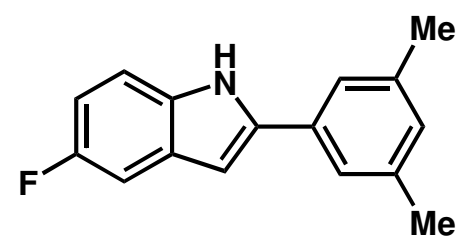

2-(3,5-Dimethyl-phenyl)-5-fluoroindole: ${ }^{1} \mathrm{H}$ NMR $\left(300 \mathrm{MHz}, \mathrm{CDCl}_{3}\right): \delta 8.29(\mathrm{~s}, 1 \mathrm{H})$, 7.26-7.31 (m, 4 H), 7.00 (broad s, 1 H), 6.94 (dt, $J=2.4,9.0 \mathrm{~Hz}, 1 \mathrm{H}), 6.76-6.77$ (m, $1 \mathrm{H}$ ). $2.41(\mathrm{~s}, 3 \mathrm{H}) .{ }^{13} \mathrm{C}$ NMR $\left(75 \mathrm{MHz}, \mathrm{CDCl}_{3}\right): \delta 160.1,157.0,140.4,139.1,133.6,132.3$, 130.2, 123.5, 111.8. IR (film, $\mathrm{cm}^{-1}$ ): 3419, 2921, 1602, 1454, 1191, 785, 675. LRMS (APCI) for $\mathrm{C}_{16} \mathrm{H}_{15} \mathrm{FN}\left(\mathrm{MH}^{+}\right)$calcd (found): 240.12 (240.28). 


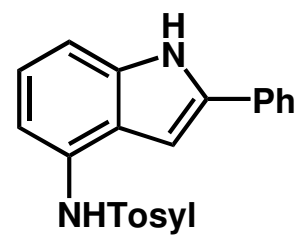

4-Methyl- $\mathrm{N}$-(2-phenyl-4-indolyl)-benzenesulfonamide: ${ }^{1} \mathrm{H} \mathrm{NMR}\left(400 \mathrm{MHz}, \mathrm{CDCl}_{3}\right)$ : $\delta 8.41(\mathrm{~s}, 1 \mathrm{H}), 7.70(\mathrm{~d}, J=6.3 \mathrm{~Hz}, 2 \mathrm{H}), 7.58(\mathrm{~d}, J=6.9 \mathrm{~Hz}, 2 \mathrm{H}), 7.43(\mathrm{t}, J=5.7 \mathrm{~Hz}, 2$ H), 7.31-7.35 (m, $1 \mathrm{H}), 7.16-7.20(\mathrm{~m}, 3 \mathrm{H}), 7.06(\mathrm{t}, J=5.7 \mathrm{~Hz}, 1 \mathrm{H}), 7.10(\mathrm{~d}, J=5.7 \mathrm{~Hz}$, $1 \mathrm{H}), 6.75(\mathrm{~s}, 1 \mathrm{H}), 6.59(\mathrm{~d}, J=0.9 \mathrm{~Hz}, 1 \mathrm{H}), 2.31$ (s, $3 \mathrm{H}) .{ }^{13} \mathrm{C}$ NMR $(75 \mathrm{MHz}$, DMSO$\left.d_{6}\right): \delta 143.7,138.8,138.2,137.7,132.8,129.3,128.5,127.5,127.4,125.1,121.7,115.1$, 109.1, 96.4, 20.3. IR (film, $\mathrm{cm}^{-1}$ ): 3321, 2972, 1698, 1478, 1159, 760. LRMS (APCI) for $\mathrm{C}_{21} \mathrm{H}_{19} \mathrm{~N}_{2} \mathrm{O}_{2} \mathrm{~S}\left(\mathrm{MH}^{+}\right)$calcd (found): 363.12 (363.36).

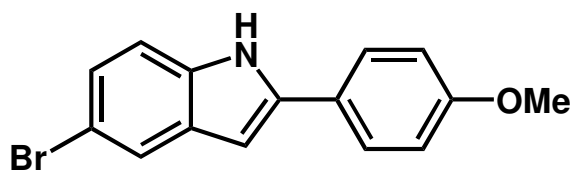

5-Bromo-2-(4-methoxy-phenyl)-indole: ${ }^{1} \mathrm{H}$ NMR (300 MHz, DMSO- $\left.d_{6}\right): \delta 11.59$ (s, 1 H), 7.75-7.78 (m, $2 \mathrm{H}), 7.64(\mathrm{~d}, J=1.8 \mathrm{~Hz}, 1 \mathrm{H}), 7.31$ (d, $J=7.6 \mathrm{~Hz}, 1 \mathrm{H}), 7.14$ (dd, $J=$ 1.8, $7.6 \mathrm{~Hz}, 1 \mathrm{H}), 7.02(\mathrm{~d}, J=8.7 \mathrm{~Hz}, 2 \mathrm{H}), 6.72(\mathrm{~d}, J=1.8 \mathrm{~Hz}, 1 \mathrm{H}), 3.80(\mathrm{~s}, 3 \mathrm{H}) .{ }^{13} \mathrm{C}$ NMR $\left(75 \mathrm{MHz}, \mathrm{DMSO}-d_{6}\right): \delta 159.8,140.1,136.4,131.5,127.4,125.1,124.2,122.5$, 115.3, 113.8, 112.6, 97.8, 56.3. IR (film, $\mathrm{cm}^{-1}$ ): 3453, 2855, 1628, 1554, 1200, 855. LRMS (APCI) for $\mathrm{C}_{15} \mathrm{H}_{13} \mathrm{BrNO}\left(\mathrm{MH}^{+}\right.$) calcd (found): 302.02, 304.02 (302.19, 304.20).

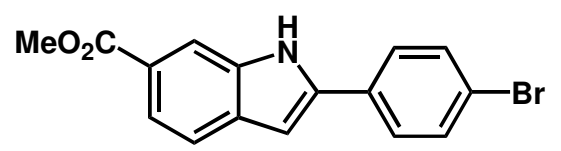

2-(4-Bromo-phenyl)-1H-indole-6-carboxylic acid methyl ester: ${ }^{1} \mathrm{H}$ NMR $(300 \mathrm{MHz}$, DMSO- $\left.d_{6}\right): \delta 11.98(\mathrm{~s}, 1 \mathrm{H}), 8.06(\mathrm{~s}, 1 \mathrm{H}), 7.85-7.88(\mathrm{~m}, 2 \mathrm{H}), 7.71-7.73(\mathrm{~m}, 2 \mathrm{H}), 7.64$ $(\mathrm{s}, 1 \mathrm{H}), 7.07(\mathrm{~d}, J=0.9 \mathrm{~Hz}, 1 \mathrm{H}), 3.89(\mathrm{~s}, 3 \mathrm{H}) .{ }^{13} \mathrm{C}$ NMR $\left(75 \mathrm{MHz}, \mathrm{DMSO}-d_{6}\right): \delta$ 168.0, 141.0, 137.3, 133.1, 132.9, 131.6, 128.3, 123.6, 122.2, 121.1, 120.9, 114.0, 100.7, 52.7. IR (film, $\mathrm{cm}^{-1}$ ): 3521, 2923, 1718, 726. LRMS (APCI) for $\mathrm{C}_{16} \mathrm{H}_{14} \mathrm{BrNO}_{2}\left(\mathrm{MH}^{+}\right)$ calcd (found): $330.01,330.11(330.15,330.25)$. 


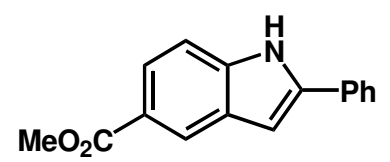

2-Phenyl-5-indole-carboxylic acid methyl ester: ${ }^{7}{ }^{1} \mathrm{H}$ NMR (300 MHz, $\left.\mathrm{CDCl}_{3}\right): \delta 8.57$ (s, $1 \mathrm{H}), 8.39$ (s, $1 \mathrm{H}), 7.91$ (dd, $J=1.8,5.4 \mathrm{~Hz}, 1 \mathrm{H}), 7.68$ (d, $J=7.5 \mathrm{~Hz}, 2 \mathrm{H}), 7.47$ (t, $J$ $=4.5 \mathrm{~Hz}, 1 \mathrm{H}), 7.34-7.43(\mathrm{~m}, 3 \mathrm{H}), 6.91(\mathrm{~d}, J=1.8 \mathrm{~Hz}, 1 \mathrm{H}), 3.91(\mathrm{~s}, 3 \mathrm{H}) .{ }^{13} \mathrm{C}$ NMR $(75$ $\left.\mathrm{MHz}, \mathrm{CDCl}_{3}\right): \delta 168.5,139.7,132.1,129.5,129.2,128.6,125.7,124.2,124.0,122.8$, 111.0, 101.4, 52.3. IR (film, $\mathrm{cm}^{-1}$ ): 3342, 1687, 755, 687. LRMS (APCI) for $\mathrm{C}_{16} \mathrm{H}_{14} \mathrm{NO}_{2}\left(\mathrm{MH}^{+}\right)$calcd (found): 252.10 (252.26).

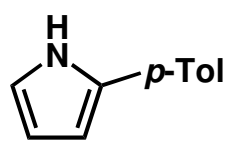

2-(4-Tolyl)-pyrrole: ${ }^{8}{ }^{1} \mathrm{H}$ NMR (300 MHz, $\left.\mathrm{CDCl}_{3}\right): \delta 8.38$ (s, $\left.1 \mathrm{H}\right), 7.38(\mathrm{~d}, J=8.1 \mathrm{~Hz}$, 2 H), 7.18 (d, $J=7.8 \mathrm{~Hz}, 2 \mathrm{H}), 6.84-6.86(\mathrm{~m}, 1 \mathrm{H}), 6.48-6.51$ (m, $1 \mathrm{H}), 6.30$ (dd, $J=2.7$, $6.0 \mathrm{~Hz}, 1 \mathrm{H}) .{ }^{13} \mathrm{C} \mathrm{NMR}\left(75 \mathrm{MHz}, \mathrm{CDCl}_{3}\right): \delta 136.3,132.7,130.4,130.0,124.3,118.8$, 110.4, 105.8, 21.5. IR (film, $\mathrm{cm}^{-1}$ ): 3435, 1707, 1507, 1362, 1225, 1120, 907, 820, 797, 734. LRMS (APCI) for $\mathrm{C}_{11} \mathrm{H}_{12} \mathrm{~N}\left(\mathrm{MH}^{+}\right)$calcd (found): 158.10 (158.19).

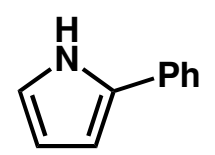

2-Phenyl-pyrrole: ${ }^{9}{ }^{1} \mathrm{H}$ NMR (300 MHz, DMSO- $\left.d_{6}\right): \delta 8.46(\mathrm{~s}, 1 \mathrm{H}), 7.51-7.54(\mathrm{~m}, 2$ H), 7.39-7.45 (m, 2 H), 7.21-7.29 (m, 1 H), 6.90-6.92 (m, $1 \mathrm{H}), 6.58-6.60(\mathrm{~m}, 1 \mathrm{H}), 6.36$ $(\mathrm{dd}, J=3.6,8.0 \mathrm{~Hz}, 1 \mathrm{H}) .{ }^{13} \mathrm{C}$ NMR $\left(75 \mathrm{MHz}\right.$, DMSO- $\left.d_{6}\right): \delta 133.2,132.5,133.2,132.5$, 129.3, 126.6, 124.3, 119.3, 110.5, 106.4. IR (film, $\mathrm{cm}^{-1}$ ): 3421, 3372, 1463, 1109, 904, 755, 725, 605. LRMS (APCI) for $\mathrm{C}_{10} \mathrm{H}_{10} \mathrm{~N}\left(\mathrm{MH}^{+}\right)$calcd (found): 144.08 (144.17).

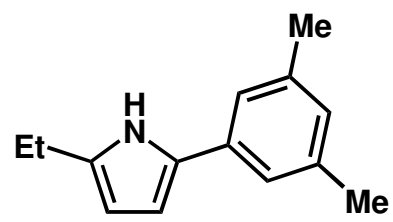

2-(3,5-Dimethyl-phenyl)-5-ethyl-1H-pyrrole: ${ }^{1} \mathrm{H}$ NMR (300 MHz, DMSO- $\left.d_{6}\right): \delta 8.13$ (s, 1 H), 7.09 (s, 2 H), 6.85 (s, 1 H), 6.42 (t, $J=3.0 \mathrm{~Hz}, 1 \mathrm{H}), 6.00$ (t, $J=2.7 \mathrm{~Hz}, 1 \mathrm{H}$ ), 
$2.72(\mathrm{q}, J=7.5 \mathrm{~Hz}, 2 \mathrm{H}), 2.37(\mathrm{~s}, 3 \mathrm{H}), 1.33(\mathrm{t}, J=7.5 \mathrm{~Hz}, 3 \mathrm{H}) .{ }^{13} \mathrm{C}$ NMR $(75 \mathrm{MHz}$, DMSO- $d_{6}$ ): $\delta 138.7,135.7,133.3,131.2,127.9,106.5,106.2,21.8,21.4,14.0$. IR (film, $\mathrm{cm}^{-1}$ ): 3404, 2968, 2916, 1602, 1503, 1322, 1193, 1036, 899, 761. LRMS (APCI) for $\mathrm{C}_{14} \mathrm{H}_{18} \mathrm{~N}\left(\mathrm{MH}^{+}\right)$calcd (found): 200.14 (200.27).

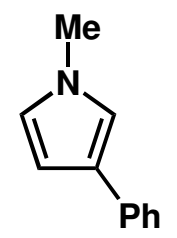

1-Methyl-3-phenyl-pyrrole: ${ }^{9-11}{ }^{1} \mathrm{H}$ NMR $\left(300 \mathrm{MHz}, \mathrm{CDCl}_{3}\right): \delta$ 7.49-7.52 (m, $\left.2 \mathrm{H}\right)$, 7.33 (t, $J=7.5 \mathrm{~Hz}, 2 \mathrm{H}), 7.13-7.18(\mathrm{~m}, 1 \mathrm{H}), 6.92(\mathrm{t}, J=2.1 \mathrm{~Hz}, 1 \mathrm{H}), 6.64(\mathrm{t}, J=2.4 \mathrm{~Hz}$, $1 \mathrm{H}), 6.45(\mathrm{t}, J=2.1 \mathrm{~Hz}, 1 \mathrm{H}), 3.71(\mathrm{~s}, 3 \mathrm{H}) .{ }^{13} \mathrm{C} \mathrm{NMR}\left(75 \mathrm{MHz}, \mathrm{CDCl}_{3}\right): \delta 136.3$, 131.0, 129.0, 125.7, 125.4, 123.1, 119.0, 106.7, 36.8. IR (film, $\mathrm{cm}^{-1}$ ): 2914, 1704, 1605, 1211. LRMS (APCI) for $\mathrm{C}_{11} \mathrm{H}_{12} \mathrm{~N}\left(\mathrm{MH}^{+}\right)$calcd (found): 158.10 (158.23). 


\section{Mechanistic Studies of Rh-Catalyzed Arylation of Free Azoles.}

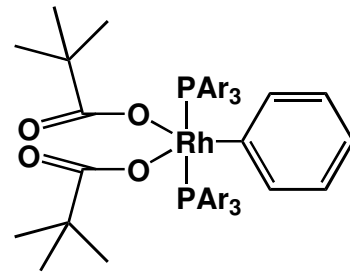

$2\left[\mathrm{Ar}=4-\left(\mathrm{CF}_{3}\right) \mathrm{C}_{6} \mathrm{H}_{4}\right]$

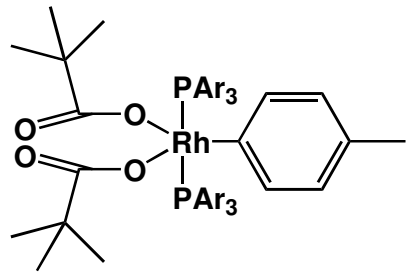

$3\left[\mathrm{Ar}=4-\left(\mathrm{CF}_{3}\right) \mathrm{C}_{6} \mathrm{H}_{4}\right]$

Synthesis of Complex 2. $\left[\mathrm{Rh}(\mathrm{coe})_{2} \mathrm{Cl}\right]_{2}(180 \mathrm{mg}, 0.25 \mathrm{mmol})$ and $\mathrm{P}[4-$ $\left.\left(\mathrm{CF}_{3}\right) \mathrm{C}_{6} \mathrm{H}_{4}\right]_{3}$ (700 mg, $\left.1.50 \mathrm{mmol}\right), \mathrm{PhI}$ (204 mg, $1.0 \mathrm{mmol}$ ), CsOPiv (930 mg, $4.0 \mathrm{mmol}$ ) and 1,4-dioxane $(5 \mathrm{~mL})$ were subsequently charged into a screw-capped vial in an argonfilled glovebox. The vial was subsequentially capped, brought out the glovebox and heated at $120{ }^{\circ} \mathrm{C}$ for $10 \mathrm{~min}$ with vigorous stirring. The resulting yellowish mixture was then cooled to room temperature, and diluted with hexanes $(15 \mathrm{ml})$. The yellow precipitation was collected on a frit, sequentially washed (hexanes/10mL×2, $\mathrm{H}_{2} \mathrm{O} / 10 \mathrm{~mL} \times 3$, hexanes/10mL×2) and then dried on high vacuum for $60 \mathrm{~min}$ to give 2 $(585 \mathrm{mg}, 89 \%)$ as a fine yellowish powder. ${ }^{1} \mathrm{H}$ NMR $\left(300 \mathrm{MHz}, \mathrm{CDCl}_{3}\right): \delta \quad 6.72-8.30$ (broad s, $24 \mathrm{H}), 6.63(\mathrm{~d}, J=7.8 \mathrm{~Hz}, 2 \mathrm{H}), 6.51(\mathrm{t}, J=7.2 \mathrm{~Hz}, 1 \mathrm{H}), 6.34(\mathrm{t}, J=7.5 \mathrm{~Hz}, 2$ $\mathrm{H}), 0.32(\mathrm{~s}, 18 \mathrm{H}) .{ }^{31} \mathrm{P}$ NMR $\left(121 \mathrm{MHz}\right.$, Dioxane- $\left.d_{8}\right): \delta 25.2\left(\mathrm{~d}, J_{\mathrm{Rh}-\mathrm{P}}=113 \mathrm{~Hz}\right) . \quad$ IR (film, $\mathrm{cm}^{-1}$ ): 1712, 1609, 1400, 1323, 1131, 1062. MS (FAB) for $\mathrm{C}_{53} \mathrm{H}_{38} \mathrm{~F}_{18} \mathrm{O}_{2} \mathrm{P}_{2} \mathrm{Rh}\left(\mathrm{M}^{+}-\right.$ OPiv) calcd (found): 1213.1 (1213.2).

Synthesis of Complex 3 was isolated in 55\% yield employing a procedure analogous to that was used for $\mathbf{2}$, with the exception that washing by hexanes was carried out at $-78{ }^{\circ} \mathrm{C}$ due to the much higher solubility of $\mathbf{3}$ in organic solvents. X-ray quality crystals were grown from chloroform/hexanes solution. ${ }^{1} \mathrm{H}$ NMR (300 MHz, Dioxane$\left.d_{8}\right): \delta 7.01-8.90(\mathrm{~m}, 24 \mathrm{H}), 6.65(\mathrm{~d}, J=8.1 \mathrm{~Hz}, 2 \mathrm{H}), 6.23(\mathrm{~d}, J=8.1 \mathrm{~Hz}, 2 \mathrm{H}), 2.09$ (s, 3 H), 0.41 (s, $18 \mathrm{H}) .{ }^{31} \mathrm{P}$ NMR (121 MHz, Dioxane- $\left.d_{8}\right): \delta 24.8\left(\mathrm{~d}, J_{\mathrm{Rh}-\mathrm{P}}=113 \mathrm{~Hz}\right) . \mathrm{IR}$ (film, $\mathrm{cm}^{-1}$ ): 1717, 1610, 1480, 1325, 1172, 1133, 1063. 


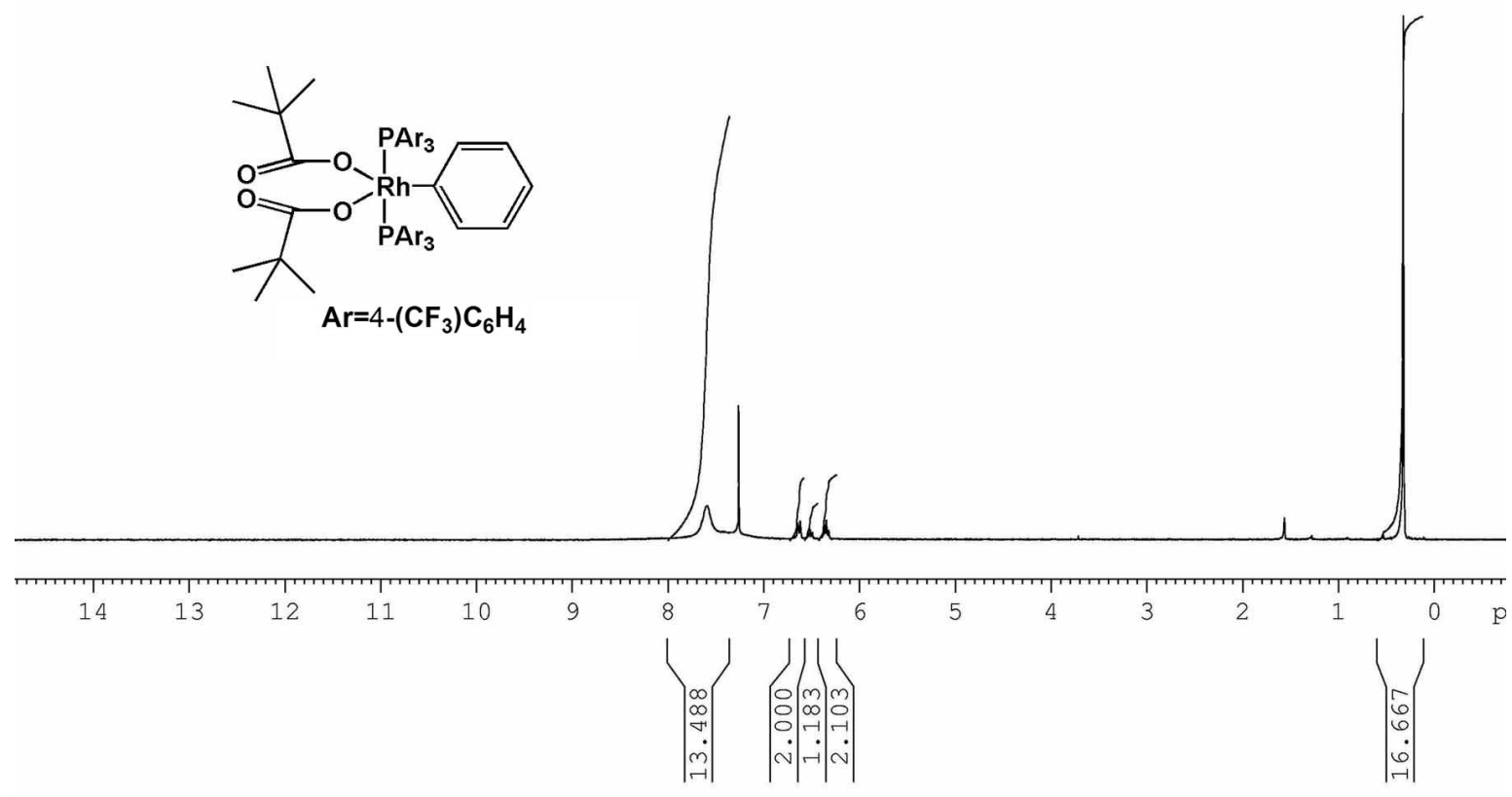

Figure S1. ${ }^{1} \mathrm{H}$ NMR $\left(\mathrm{CDCl}_{3}\right)$ of $\mathrm{Rh}(\mathrm{OPiv})_{2}\left\{\mathrm{P}\left[4-\left(\mathrm{CF}_{3}\right) \mathrm{C}_{6} \mathrm{H}_{4}\right]_{3}\right\}_{2} \mathrm{Ph}(\mathbf{2})$.

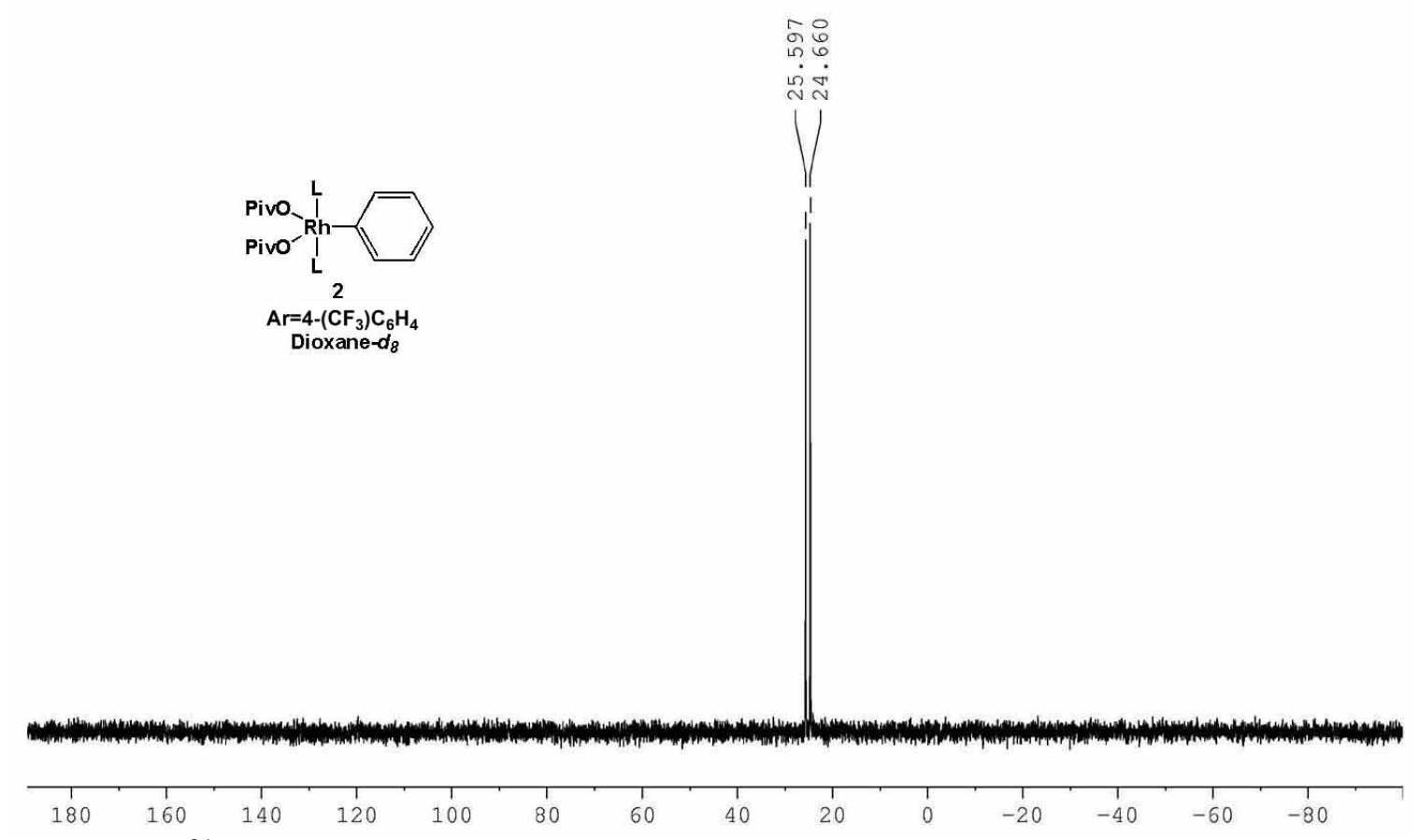

Figure S2. ${ }^{31} \mathrm{P}$ NMR of $\mathrm{Rh}(\mathrm{OPiv})_{2}\left\{\mathrm{P}\left[4-\left(\mathrm{CF}_{3}\right) \mathrm{C}_{6} \mathrm{H}_{4}\right]_{3}\right\}_{2} \mathrm{Ph}(2)$ in dioxane- $d_{8}$. 


\section{NMR Monitoring of Catalytic Reaction}

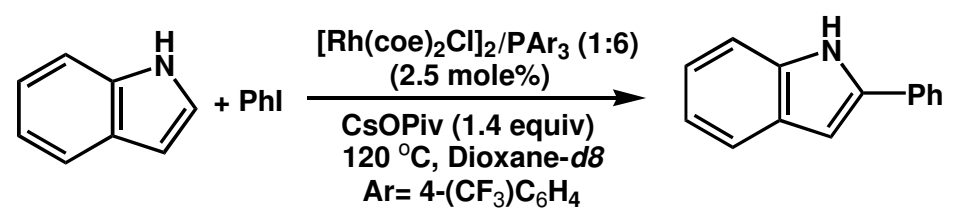

The above reaction $\left([\right.$ Indole $]=0.50 \mathrm{M},[\mathrm{PhI}]=0.60 \mathrm{M},[1]=25 \mathrm{mM},[\mathrm{phos}]_{\text {total }}=150 \mathrm{mM}$ ) was carried out in an argon-filled J-Young NMR tube at $120^{\circ} \mathrm{C}$ in dioxane- $d_{8} .{ }^{31} \mathrm{P}$ NMR was taken at room temperature.

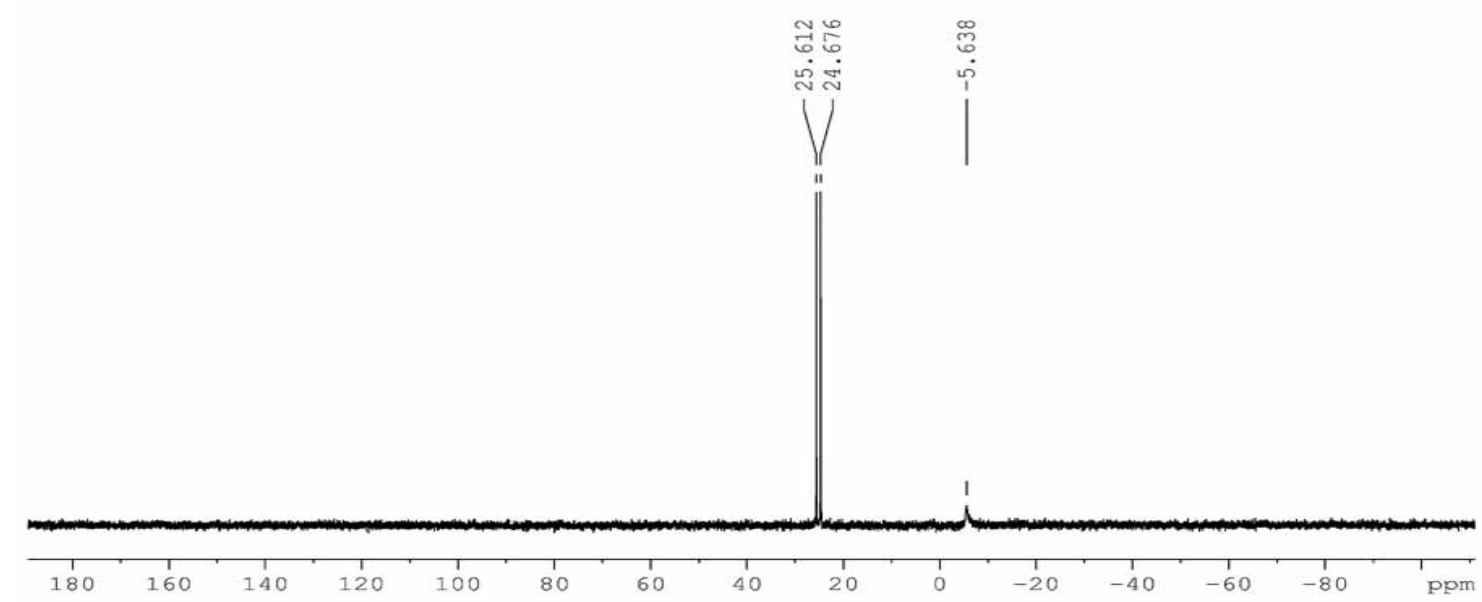

Figure S3. ${ }^{31} \mathrm{P}$ NMR of catalytic reaction mixture taken after $30 \mathrm{~min}$ of heating, dioxane$d_{8}$. Free phosphine $\mathbf{L}(\delta-5.64 \mathrm{ppm})$

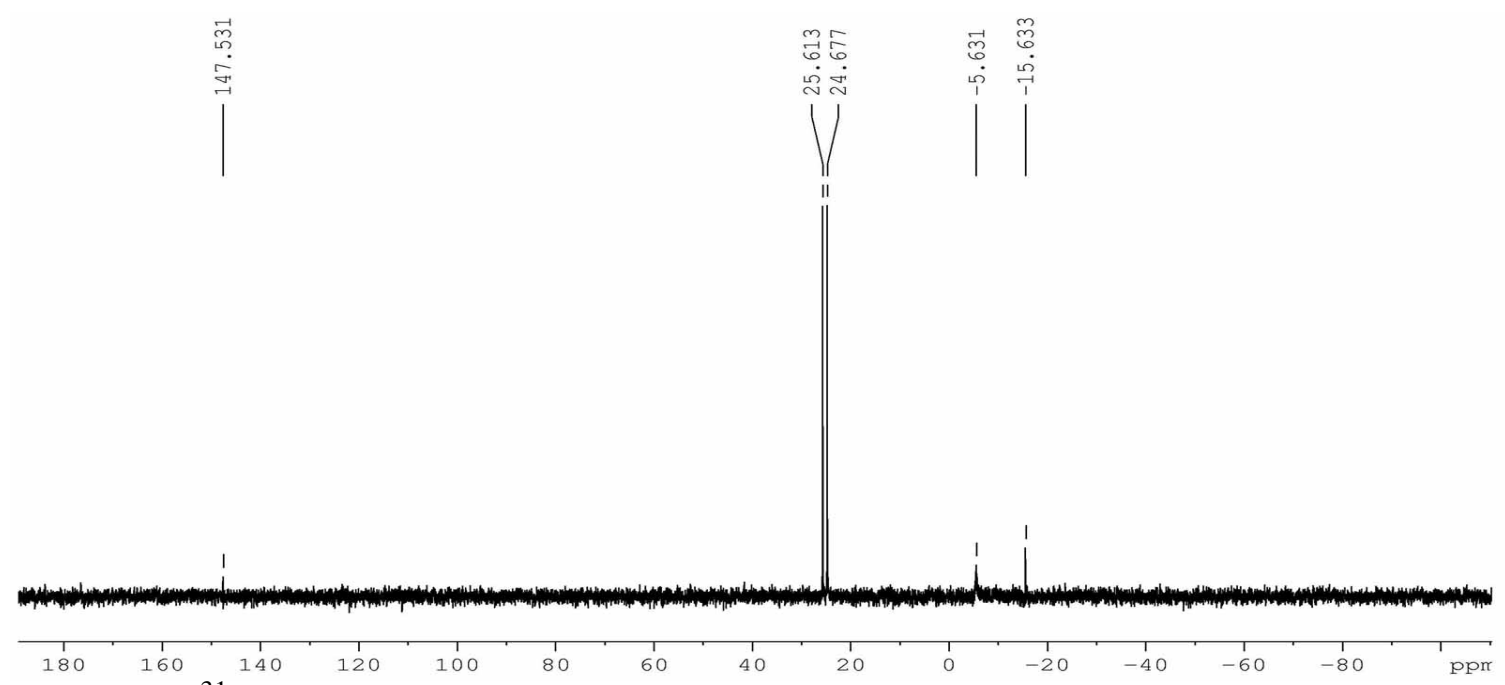

Figure S4. ${ }^{31} \mathrm{P}$ NMR of catalytic reaction mixture taken after $8 \mathrm{~h}$ of heating in dioxane- $d_{8}$.

Although the decomposition of phosphine is evident after prolonged heating, complex 2 remained as the only observable $\mathrm{Rh}$-phosphine species (rhodium phosphine couping). 


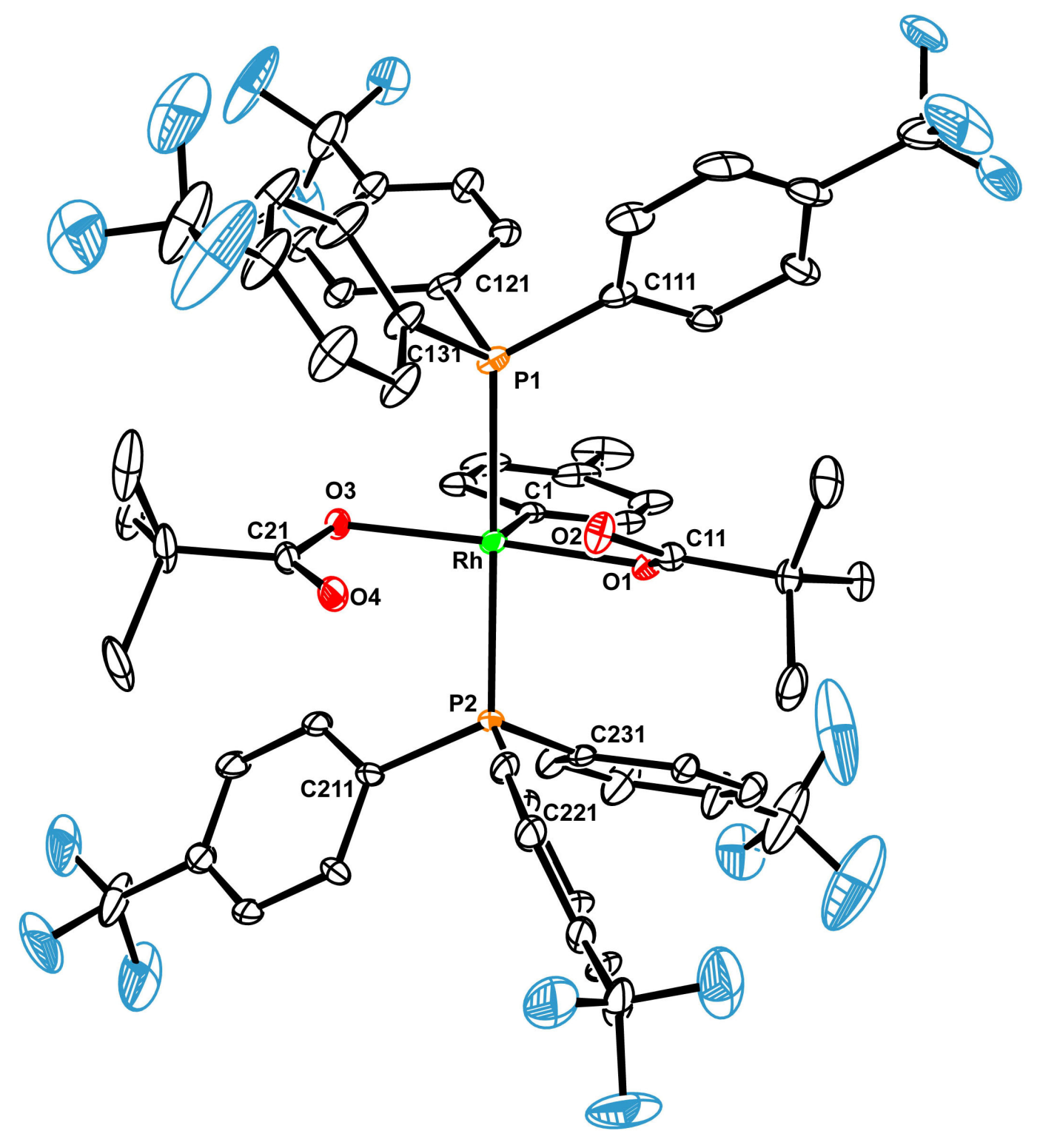

Ortep Drawing of 3. A molecule of solvent and conformation isomers for fluroines were omitted. For selected bond length and angles see table $2 \& 3$. For complete data see the CIF file. 
Table 2. Selected Bond Lengths (Angstroms) for $\mathbf{3}$

\begin{tabular}{|l|llr|llc|}
\hline Atom & A & B & Dist & A & B & Dist \\
\hline & O1 & C11 & 1.298 & P2 & Rh & 2.346 \\
& O1 & Rh & 2.034 & Rh & C1 & 1.989 \\
& O2 & C11 & 1.226 & Rh & O1 & 2.034 \\
& O3 & C21 & 1.288 & Rh & O3 & 2.055 \\
& O3 & Rh & 2.055 & Rh & P2 & 2.346 \\
& O4 & C21 & 1.218 & Rh & P1 & 2.357 \\
& P1 & Rh & 2.357 & & & \\
\hline
\end{tabular}

Table 3. Selected Bond Angles for $\mathbf{3}$

\begin{tabular}{|c|c|c|c|c|c|c|c|c|}
\hline \multirow[t]{17}{*}{ Atom } & $\bar{A}$ & $\mathrm{~B}$ & $\bar{C}$ & Angle & $\bar{A}$ & $\bar{B}$ & $\bar{C}$ & Angle \\
\hline & $\overline{\mathrm{C} 2}$ & $\mathrm{C} 1$ & $\mathrm{Rh}$ & 122.61 & C231 & $\mathrm{P} 2$ & C221 & 104.46 \\
\hline & C6 & C1 & $\mathrm{Rh}$ & 120.73 & C231 & P2 & C211 & 103.88 \\
\hline & $\mathrm{O} 2$ & C11 & O1 & 120.24 & C231 & P2 & $\mathrm{Rh}$ & 112.85 \\
\hline & $\mathrm{O} 2$ & C11 & C12 & 123.02 & C221 & P2 & C211 & 104.81 \\
\hline & O1 & C11 & C12 & 116.74 & C221 & P2 & $\mathrm{Rh}$ & 112.67 \\
\hline & O4 & C21 & O3 & 121.9 & C211 & P2 & $\mathrm{Rh}$ & 116.96 \\
\hline & O4 & C21 & C22 & 121.58 & C1 & $\mathrm{Rh}$ & O1 & 89.31 \\
\hline & O3 & C21 & C22 & 116.48 & C1 & $\mathrm{Rh}$ & O3 & 92.81 \\
\hline & C11 & O1 & Rh & 111.83 & C1 & $\mathrm{Rh}$ & P2 & 91.89 \\
\hline & C21 & O3 & $\mathrm{Rh}$ & 105.5 & C1 & $\mathrm{Rh}$ & P1 & 90.58 \\
\hline & C111 & P1 & C121 & 103.53 & O1 & $\mathrm{Rh}$ & O3 & 177.25 \\
\hline & C111 & P1 & C131 & 103.49 & O1 & $\mathrm{Rh}$ & P2 & 84.39 \\
\hline & C111 & P1 & $\mathrm{Rh}$ & 119 & O1 & $\mathrm{Rh}$ & P1 & 95.72 \\
\hline & C121 & P1 & C131 & 103.42 & O3 & $\mathrm{Rh}$ & P2 & 93.79 \\
\hline & C121 & P1 & Rh & 112.77 & O3 & $\mathrm{Rh}$ & P1 & 86.01 \\
\hline & C131 & P1 & $\mathrm{Rh}$ & 112.98 & P2 & $\mathrm{Rh}$ & P1 & 177.53 \\
\hline
\end{tabular}


Kinetics of Stoichiometric Reactions.

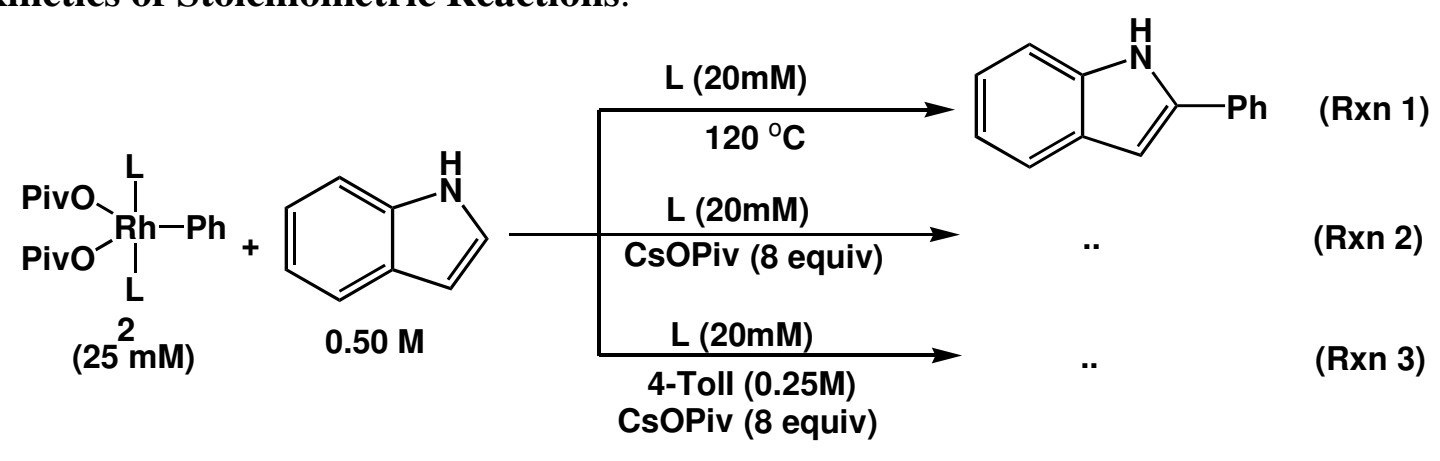

A stock solution (Stock A) was prepared by mixing the following solutions: L (1.0 $\mathrm{mL}, 1.0 \mathrm{M}$ in 1,4-dioxane), indole (16.0 mL, 1.25 $\mathrm{M}$ in 1,4-dioxane) and dodecane (1.60 $\mathrm{mL}, 72.5 \mathrm{mg} / \mathrm{mL}$ ) and then was diluted by dioxane to a total volume of $20.0 \mathrm{~mL}$.

For reaction 1: A reaction vial was charged with $2(16.4 \mathrm{mg}, 12.5 \mu \mathrm{mol})$, stock A $(250 \mu \mathrm{L})$ and dioxane $(250 \mu \mathrm{L})$. The vial was capped with a Teflon septum-equipped cap, heated at $120^{\circ} \mathrm{C}$ for 3 minutes and the reaction was then monitored by GC-MS.

For reaction 2: A reaction vial was charged with $2(16.4 \mathrm{mg}, 12.5 \mu \mathrm{mol})$, stock A $(250 \mu \mathrm{L}), \mathrm{CsOPiv}(23 \mathrm{mg}, 0.10 \mathrm{mmol})$ and dioxane $(250 \mu \mathrm{L})$. The vial was capped with a Teflon septum-equipped cap, heated at $120{ }^{\circ} \mathrm{C}$ for 3 minutes and the reaction was then monitored by GC-MS.

For reaction 3: A reaction vial was charged with $2(16.4 \mathrm{mg}, 12.5 \mu \mathrm{mol})$, stock A $(250 \mu \mathrm{L}), 4-T o l I ~(100 \mu \mathrm{L}, 1.25 \mathrm{M})$ and dioxane $(150 \mu \mathrm{L})$. The vial was capped with a Teflon septum-equipped cap, heated at $120{ }^{\circ} \mathrm{C}$ for 3 minutes and the reaction was then monitored by GC-MS. Each of the above experiments was performed twice (Figure S5). 


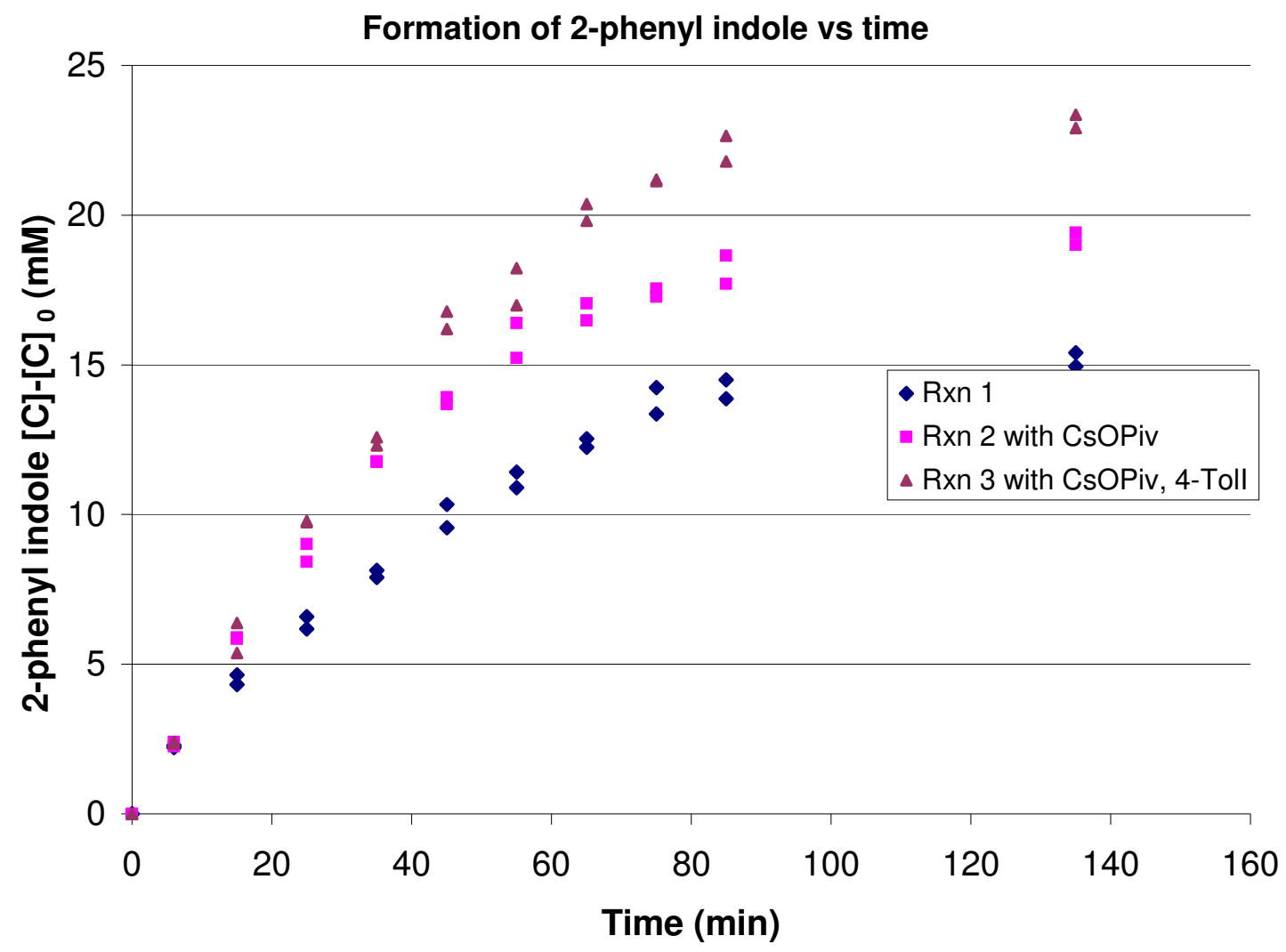

Figure S5. Stoichiometric Reaction of 2 and Indole.

Initial conditions:

Rxn 1: [2] $=25 \mathrm{mM}$, [indole] $=0.5 \mathrm{M},[\mathbf{L}]=25 \mathrm{mM}$. $^{1}$

Rxn 2: [2] $=25 \mathrm{mM}$, [indole] $=0.5 \mathrm{M},[\mathbf{L}]=25 \mathrm{mM}$, CsOPiv (heterogeneous, 8 equiv)

Rxn 3: [2] $=25 \mathrm{mM}$, [indole] $=0.5 \mathrm{M},[\mathbf{L}]=25 \mathrm{mM}$, CsOPiv (heterogeneous, 8 equiv), [4$\mathrm{TolI}]=0.25 \mathrm{M}^{2}$

Initial rate for the formation of 2-phenyl indole(first 300 seconds)

Rxn 1=0.375(7) $\mathrm{mM}^{*} \min ^{-1}$.

$\operatorname{Rxn} 2=0.387(9) \mathrm{mM}^{*} \min ^{-1}$

Rxn 3=0.397(5) $\mathrm{mM}^{*} \min ^{-1}$

${ }^{1} 1$ equivalence of phosphine $\mathbf{L}$ was added to maintain the concentration of free phosphine.

2 Formation of 2-(4-Tolyl) indole was also observed after 30 minutes via the generation of complex 3 . 


\section{Determination of Reaction Order Based on Initial Rate Dependence on Reagent Concentration: ${ }^{3}$}

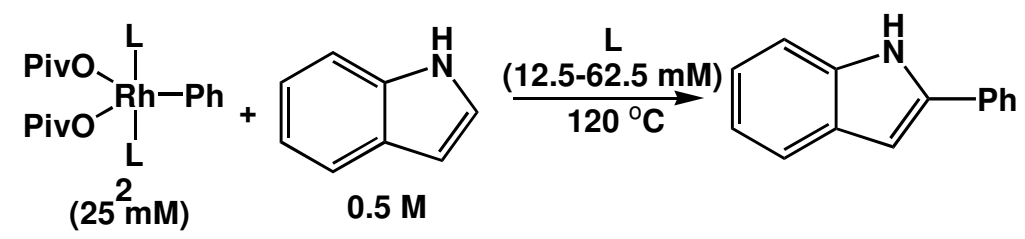

\section{Rate dependence on phosphine [L] (12.5-65.2 mM):}

Stock solution B was prepared as following: Indole $(2.40 \mathrm{~mL}, 1.25 \mathrm{M}$ in dioxane), dodecane $(240 \mu \mathrm{L}, 72.5 \mathrm{mg} / \mathrm{mL})$ and dioxane (diluted to a total amount of $3.0 \mathrm{~mL}$ )

Five different amounts of $\mathbf{L}(25.0 \mu \mathrm{L}, 33.5 \mu \mathrm{L}, 50.0 \mu \mathrm{L}, 61.5 \mu \mathrm{L}, 135.5 \mu \mathrm{L}, 0.50 \mathrm{M}$ in dioxane), $2(16.4 \mathrm{mg}, 12.5 \mu \mathrm{mol})$, stock B $(250 \mu \mathrm{L})$ and five different amounts of dioxane $(225 \mu \mathrm{L}, 216 \mu \mathrm{L}, 200 \mu \mathrm{L}, 188 \mu \mathrm{L}, 114 \mu \mathrm{L})$ were charged into 5 reaction vials, respectively. Vials were capped and heated at $120{ }^{\circ} \mathrm{C}$. Monitoring starts after $3 \mathrm{~min}$ of preheating and initial rates recorded for first 300 seconds $(5$ minutes, conversion $<10 \%)$. Experiments were repeated once. Data are plotted in Figure S6.

\begin{tabular}{|l|r|r|r|r|r|}
\hline LL] $(\mathrm{mM})$ & 12.5 & 16.7 & 25 & 30.7 & 65.2 \\
\hline $1 /[\mathrm{L}]\left(\mathrm{mM}^{-1}\right)$ & 80.0 & 59.9 & 40.0 & 32.6 & 15.3 \\
\hline Initial rate $\left(\mathrm{mM}^{*} \mathrm{~min}^{-1}\right)$ & 0.803 & 0.658 & 0.398 & 0.324 & 0.149 \\
\hline Initial rate $\left(\mathrm{mM}^{*} \mathrm{~min}^{-1}\right)$ & 0.825 & 0.607 & 0.367 & 0.358 & 0.133 \\
\hline
\end{tabular}

Conditions: [indole] $=0.50 \mathrm{M},[2]=25 \mathrm{mM}$, [L] $(12.5-65.2 \mathrm{mM})$.

Initial rate vs $1 /[\mathrm{L}]$

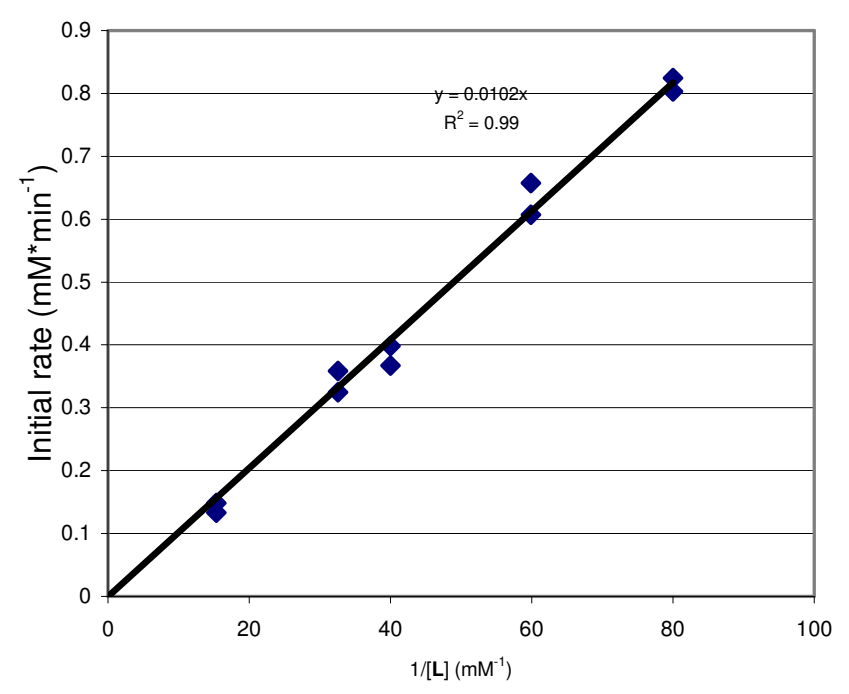

Figure S6. Initial Rates-Inversed-first order plot on phosphine [L]

\footnotetext{
${ }^{3}$ Due to the decomposition of 2 in the absence of CsOPiv and PhI (trapping effect), reaction order for the stoichiometric reaction of $\mathbf{2}$ and indole was determined using initial rate kinetics.
} 


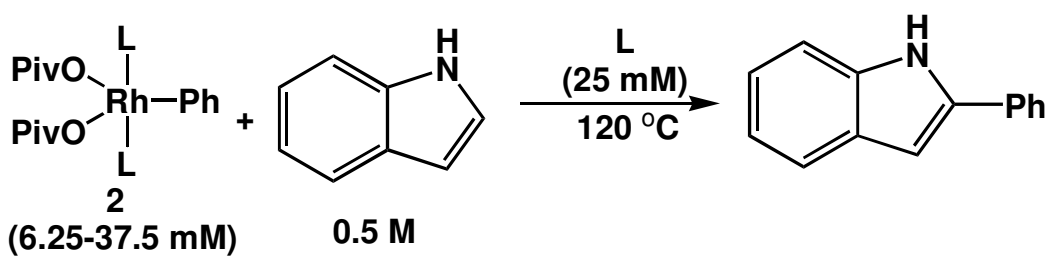

\section{Rate dependence on [2] (6.25-37.5mM):}

Stock solution C: Indole (2.4 mL, 1.25 M in dioxane), dodecane $(240 \mu \mathrm{L}, 72.5 \mathrm{mg} /$ $\mathrm{mL}), \mathbf{L}(150.0 \mu \mathrm{L}, 1.0 \mathrm{M}$ in dioxane) and dioxane (diluted to a total amount of $3.0 \mathrm{~mL})$.

Five different amount of 2 (4.1 mg, $8.2 \mathrm{mg}, 16.4 \mathrm{mg}, 20.5 \mathrm{mg}, 24.6 \mathrm{mg})$, stock C (250 $\mu \mathrm{L})$ and dioxane $(250 \mu \mathrm{L})$ were charged into five reaction vials, respectively. Vials were capped and heated at $120{ }^{\circ} \mathrm{C}$. Monitoring starts after $3 \mathrm{~min}$ of preheating and initial rates recorded for first 300 seconds ( 5 minutes, conversion $<10 \%$ ). Experiments were repeated once. Data are plotted in Figure S7.

\begin{tabular}{|l|r|r|r|r|r|}
\hline$[2](\mathrm{mM})$ & 6.25 & 12.5 & 25 & 31.25 & 37.5 \\
\hline Initial rate $\left(\mathrm{mM}^{*} \mathrm{~min}^{-1}\right)$ & 0.064 & 0.178 & 0.373 & 0.451 & 0.559 \\
\hline Initial rate $\left(\mathrm{mM}^{*} \mathrm{~min}^{-1}\right)$ & 0.070 & 0.169 & 0.385 & 0.432 & 0.517 \\
\hline
\end{tabular}

Conditions: $[$ indole $]=0.50 \mathrm{M},[\mathbf{L}]=25 \mathrm{mM}$.

Initial rate vs [2]

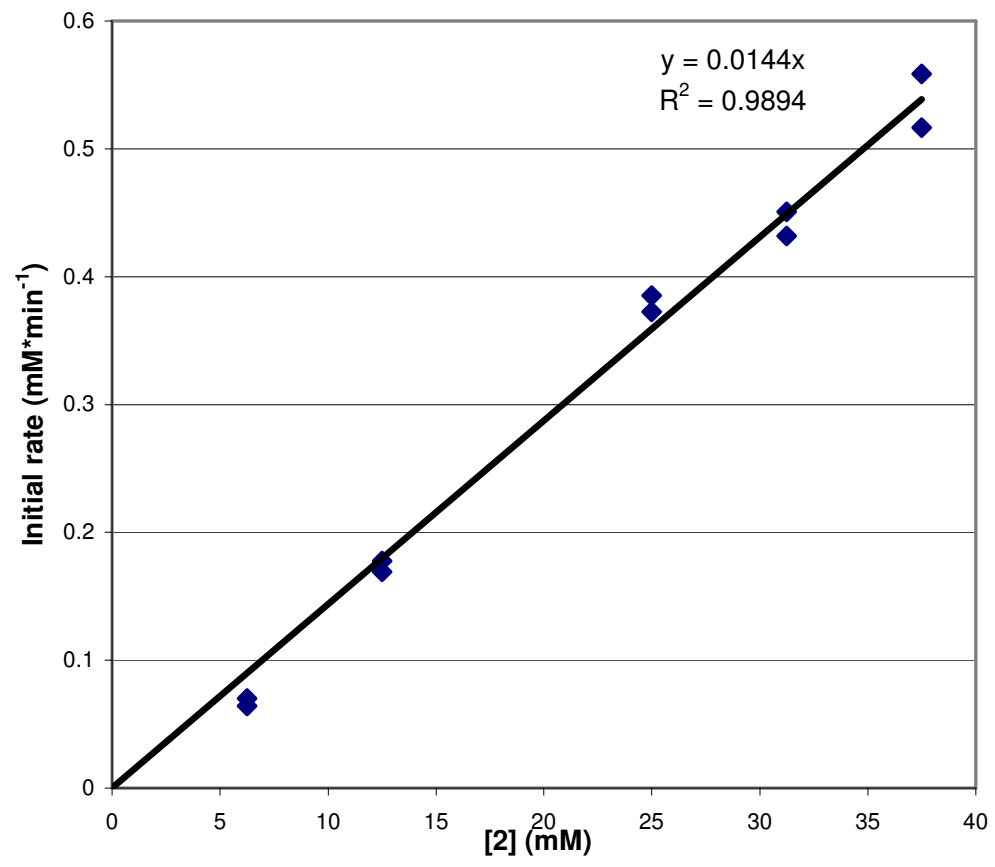

Figure S7. First order plot on [2] 


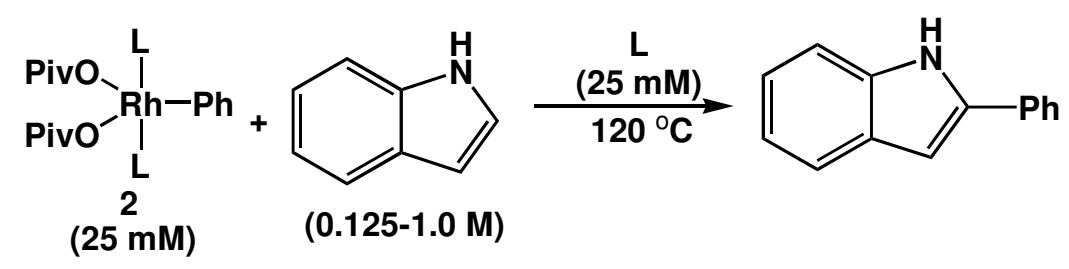

\section{Rate dependence on [indole] (0.125-1.0 M):}

Stock solution D: $\mathbf{L}(75.0 \mu \mathrm{L}, 1.0 \mathrm{M}$ in dioxane), dodecane $(120 \mu \mathrm{L}, 72.5 \mathrm{mg} / \mathrm{mL})$ and dioxane (diluted to a total amount of $3.00 \mathrm{~mL}$ ).

Five different amounts of indole $(50.0 \mu \mathrm{L}, 100 \mu \mathrm{L}, 200 \mu \mathrm{L}, 300 \mu \mathrm{L}, 400 \mu \mathrm{L}, 1.25 \mathrm{M}$ in dioxane), $2(16.4 \mathrm{mg}, 12.5 \mu \mathrm{mol})$, stock $\mathrm{D}(50 \mu \mathrm{L})$ and five different amounts of dioxane $(400 \mu \mathrm{L}, 350 \mu \mathrm{L}, 250 \mu \mathrm{L}, 150 \mu \mathrm{L}, 50 \mu \mathrm{L})$ were charged into 5 reaction vials, separately. Vials were capped and heated at $120{ }^{\circ} \mathrm{C}$. Monitoring starts after $3 \mathrm{~min}$ of preheating and initial rates recorded for first 300 seconds ( 5 minutes, conversion $<10 \%$ ). Experiments were repeated once. Data are plotted in Figure S8.

\begin{tabular}{|l|r|r|r|r|r|}
\hline Indole] $(\mathrm{M})$ & 0.125 & 0.25 & 0.5 & 0.75 & 1.0 \\
\hline Initial rate $\left(\mathrm{mM}^{*} \mathrm{~min}^{-1}\right)$ & 0.117 & 0.233 & 0.362 & 0.567 & 0.719 \\
\hline Initial rate $\left(\mathrm{mM}^{*} \mathrm{~min}^{-1}\right)$ & 0.120 & 0.195 & 0.394 & 0.588 & 0.755 \\
\hline
\end{tabular}

Conditions: $[\mathbf{2}]=25.0 \mathrm{mM},[\mathbf{L}]=25.0 \mathrm{mM}$.

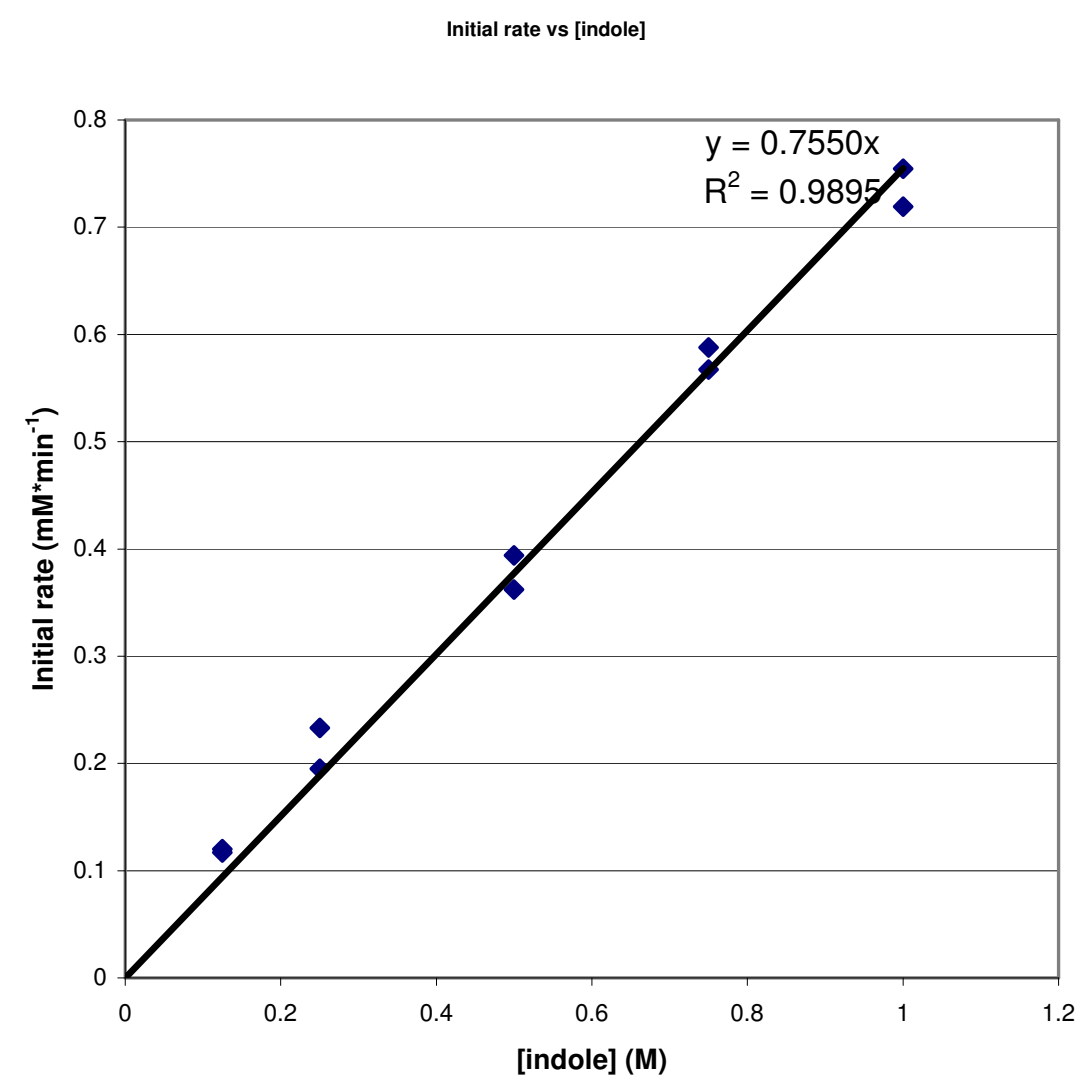

Figure S8. First Order Plot on [Indole]. 


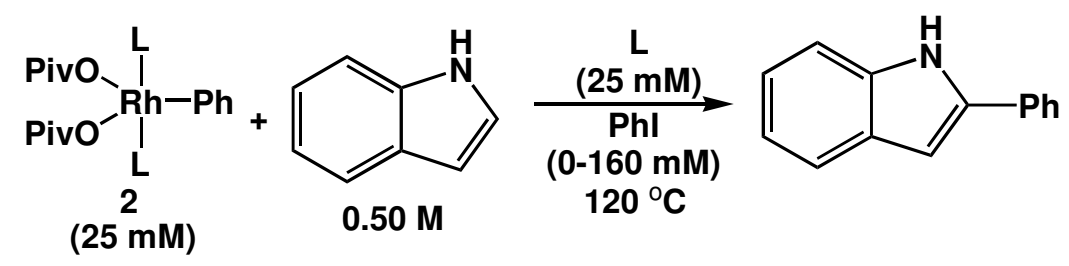

\section{Rate dependence on $[\mathrm{PhI}](0-160 \mathrm{mM})$ :}

Five different amounts of PhI ( $0 \mu \mathrm{L}, 20 \mu \mathrm{L}, 40 \mu \mathrm{L}, 80 \mu \mathrm{L}, 160 \mu \mathrm{L}, 1.0 \mathrm{M}$ in dioxane), 2 $(16.4 \mathrm{mg}, 12.5 \mu \mathrm{mol})$, stock A $(250 \mu \mathrm{L})$ and five different amounts of dioxane $(250 \mu \mathrm{L}$, $230 \mu \mathrm{L}, 210 \mu \mathrm{L}, 170 \mu \mathrm{L}, 90 \mu \mathrm{L}$ ) were charged into 5 reaction vials, separately. Vials were capped and heated at $120{ }^{\circ} \mathrm{C}$. Monitoring starts after $3 \mathrm{~min}$ of preheating and initial rates recorded for first 300 seconds ( 5 minutes, conversion $<10 \%$ ). Experiments were performed once due to apparent zero order dependence on $[\mathrm{PhI}]$. Data plotted in Figure S9.

\begin{tabular}{|l|c|c|c|c|c|}
\hline$[\mathrm{Phl}](\mathrm{mM})$ & 0 & 20 & 40 & 80 & 160 \\
\hline Initial rate $\left(\mathrm{mM}^{*} \mathrm{~min}^{-1}\right)$ & 0.380 & 0.414 & 0.399 & 0.382 & 0.386 \\
\hline
\end{tabular}

Conditions: $[$ indole $]=0.50 \mathrm{M},[2]=25.0 \mathrm{mM},[\mathbf{L}]=25.0 \mathrm{mM}$.

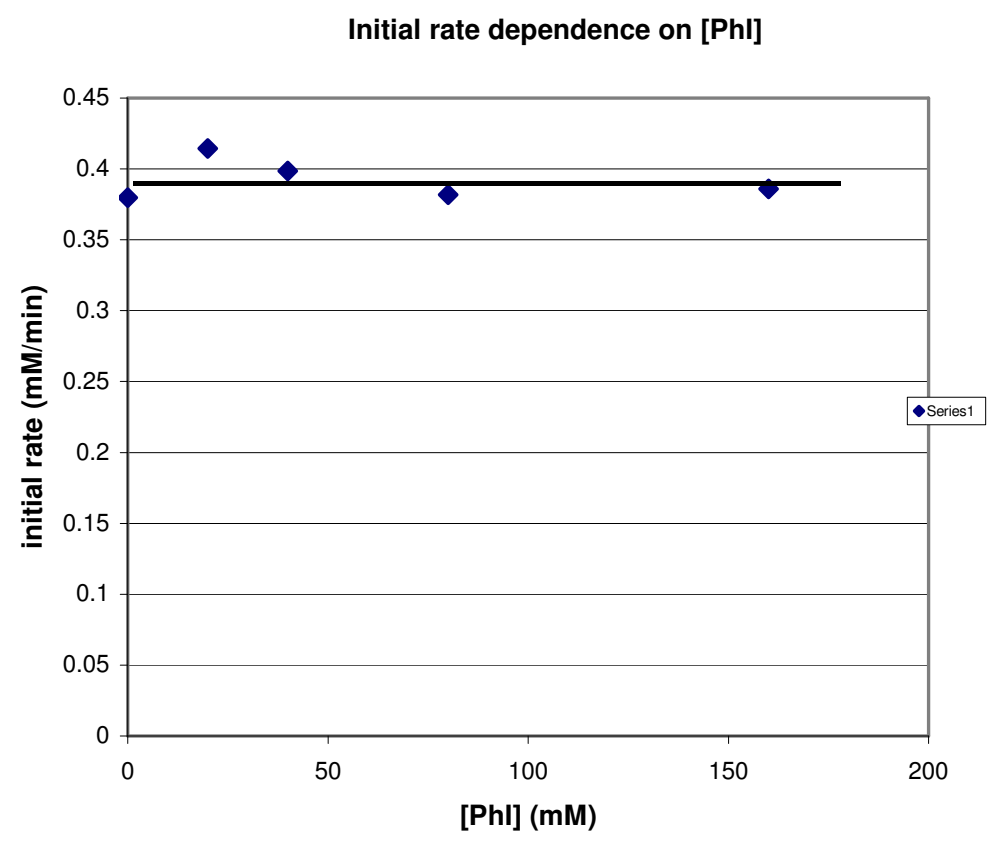

Figure S9.Zero order Plot on $[\mathrm{PhI}]$ 


\section{Kinetics of Catalytic Reactions ${ }^{4}$}

Measurement of H/D kinetic isotope effect: In an argon filled glovebox, 1 (9.0 $\mathrm{mg}, 0.0125 \mathrm{mmol}), \mathrm{P}\left[4-\left(\mathrm{CF}_{3}\right) \mathrm{C}_{6} \mathrm{H}_{4}\right]_{3}(\mathbf{L}, 36.0 \mathrm{mg}, 0.075 \mathrm{mmol})$, indole $(58.5 \mathrm{mg}, 0.50$ mmol), benzophenone (internal standard, $29 \mathrm{mg}$ ), CsOPiv (164 mg, $0.70 \mathrm{mmol}$ ) and $\mathrm{PhI}$ (122 $\mathrm{mg}, 0.60 \mathrm{mmol}$ ) was charged into a vial, followed by the addition of 1,4-dioxane $(1.0 \mathrm{~mL})$. Similarly, reaction mixture were loaded into three other vials with 1 -indole- $d_{1}$ $(59.0 \mathrm{mg}, 0.50 \mathrm{mmol}), 2$-indole- $d_{l}(59.0 \mathrm{mg}, 0.50 \mathrm{mmol})$ and 3 -indole- $d_{l}(59.0 \mathrm{mg}, 0.50$ mmol), respectively. The vials were capped, brought out the glovebox, and heated at 120 ${ }^{\circ} \mathrm{C}$ in a reaction block. After 5 minutes of preheating, reaction samples were taken periodically via syringe and their component concentration was analyzed via a GC/FID. H/D scrambling of indole was checked via GC/MS detector separately. Data are plotted in Figure S10. Initial rates are plotted in Figure S11.

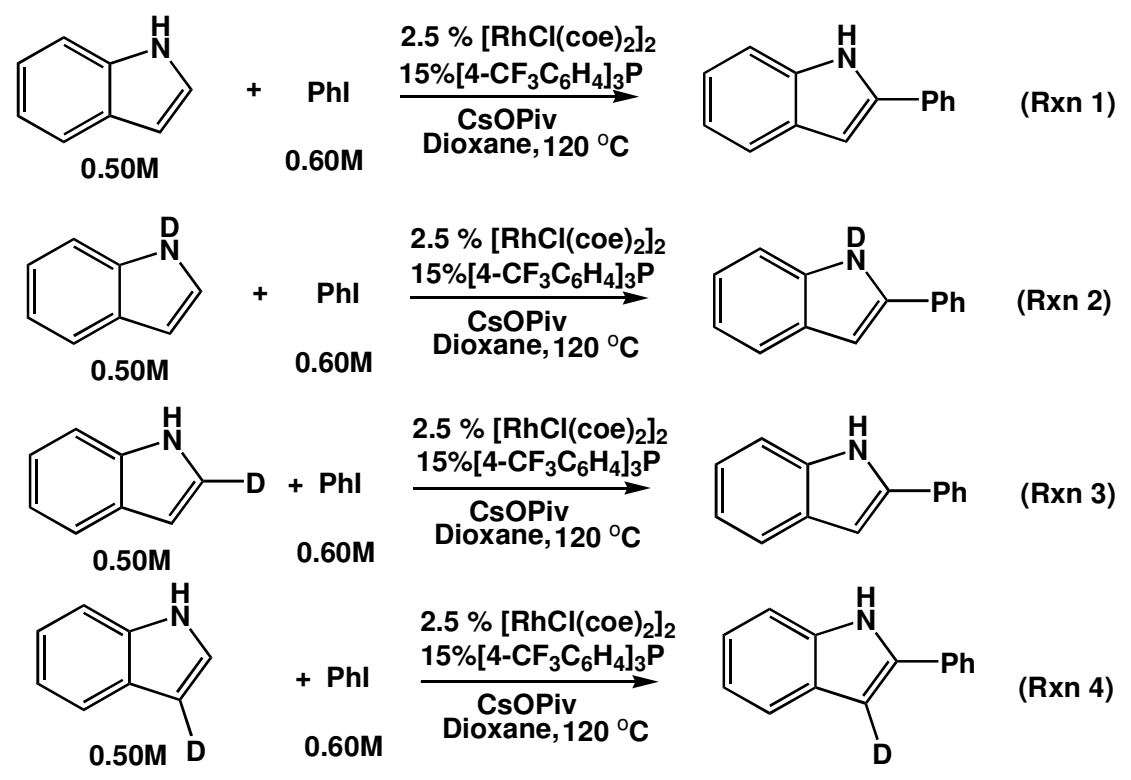

\footnotetext{
${ }^{4}$ Due to the gradual decomposition of phosphine (erosion) during reaction process, direct measurements of $\mathrm{k}_{\mathrm{H}}$ and $\mathrm{k}_{\mathrm{D}}$ from individual reactions were not feasible. Initial rate kinetics were applied instead.
} 
Reaction of Isotope-Incorporated Indole with PhI at Initial Stage

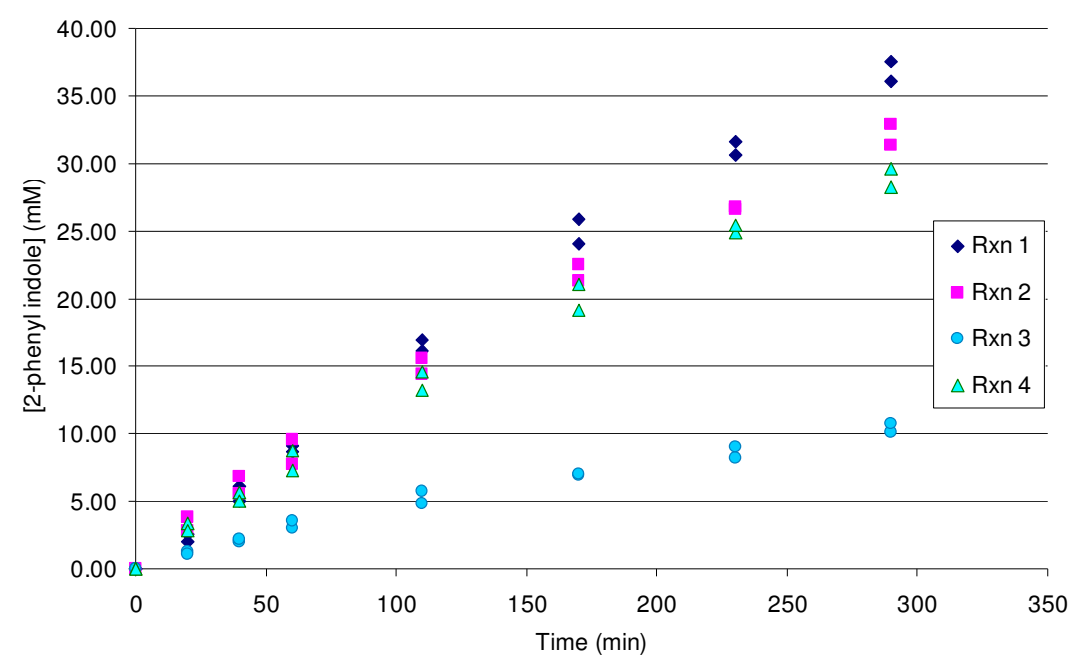

Figure S10. Initial conditions: $[\mathbf{1}]=12.5 \mu \mathrm{M},[\mathbf{L}]=37.5 \mu \mathrm{M}$, [Indole $]=0.50 \mathrm{M},[\mathrm{PhI}]=$ $0.60 \mathrm{M}$.

Rxn 1

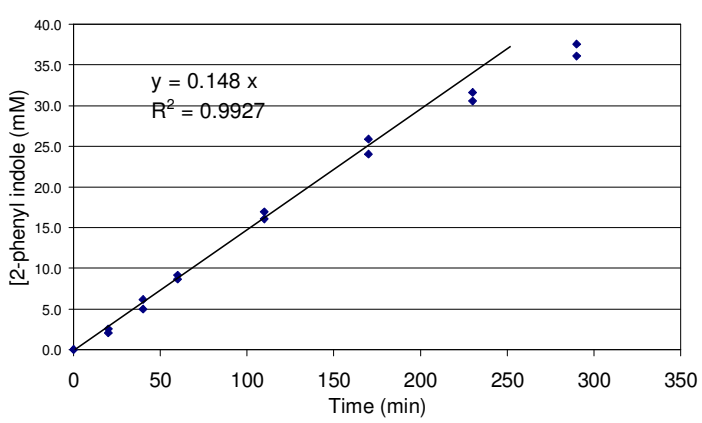

$\operatorname{Rxn} 3$

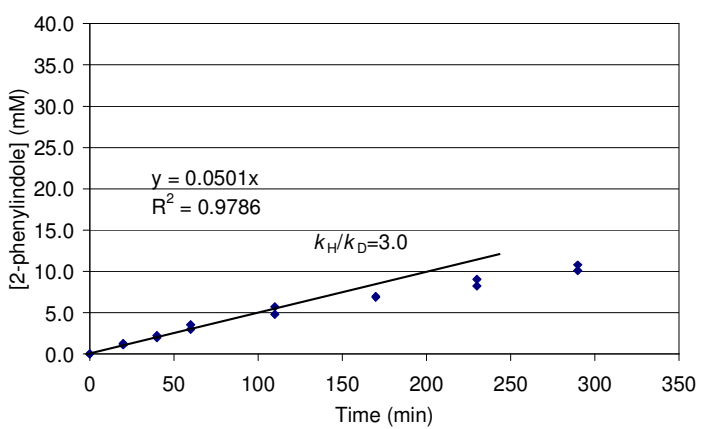

Figure S11

D/H scrambling was monitored by GC/MS separately. 1-indole- $d_{1}$ lost $\sim 20 \%$ deuterium after $200 \mathrm{~min}, 2$-indole- $d_{1}$ lost $<5 \%$ deuterium after $200 \mathrm{~min}$, 3-indole- $d_{1}$ lost $\sim 30 \%$ deuterium in 200 minutes. Partial loss of deuterium from 1-indole- $d_{1}$ and 3-indole- $d_{1}$ does not cause significant deviation in KIE measurement, as KIE at both positions are close to $1\left(k_{\mathrm{D}} \approx k_{\mathrm{H}}\right)$. 


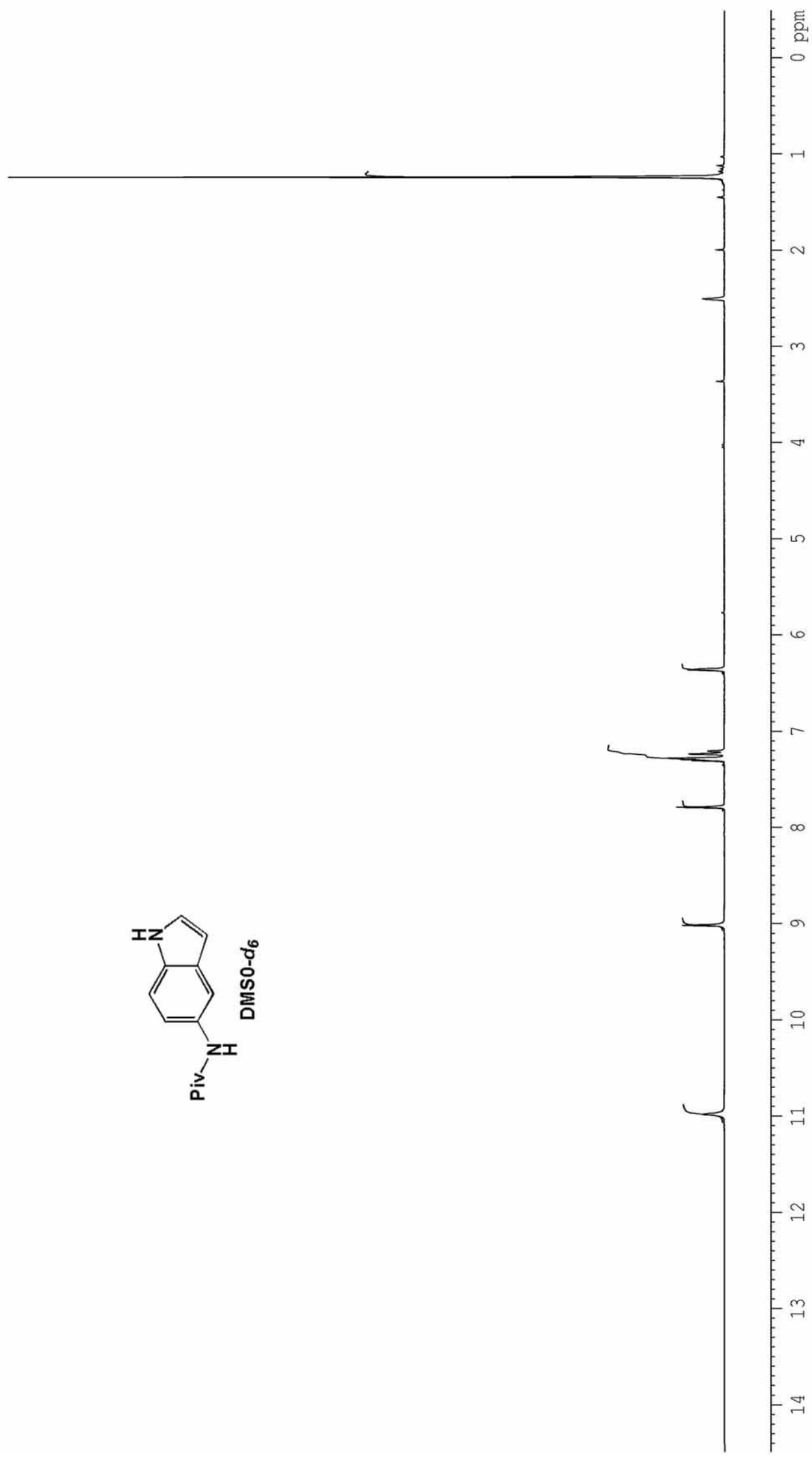




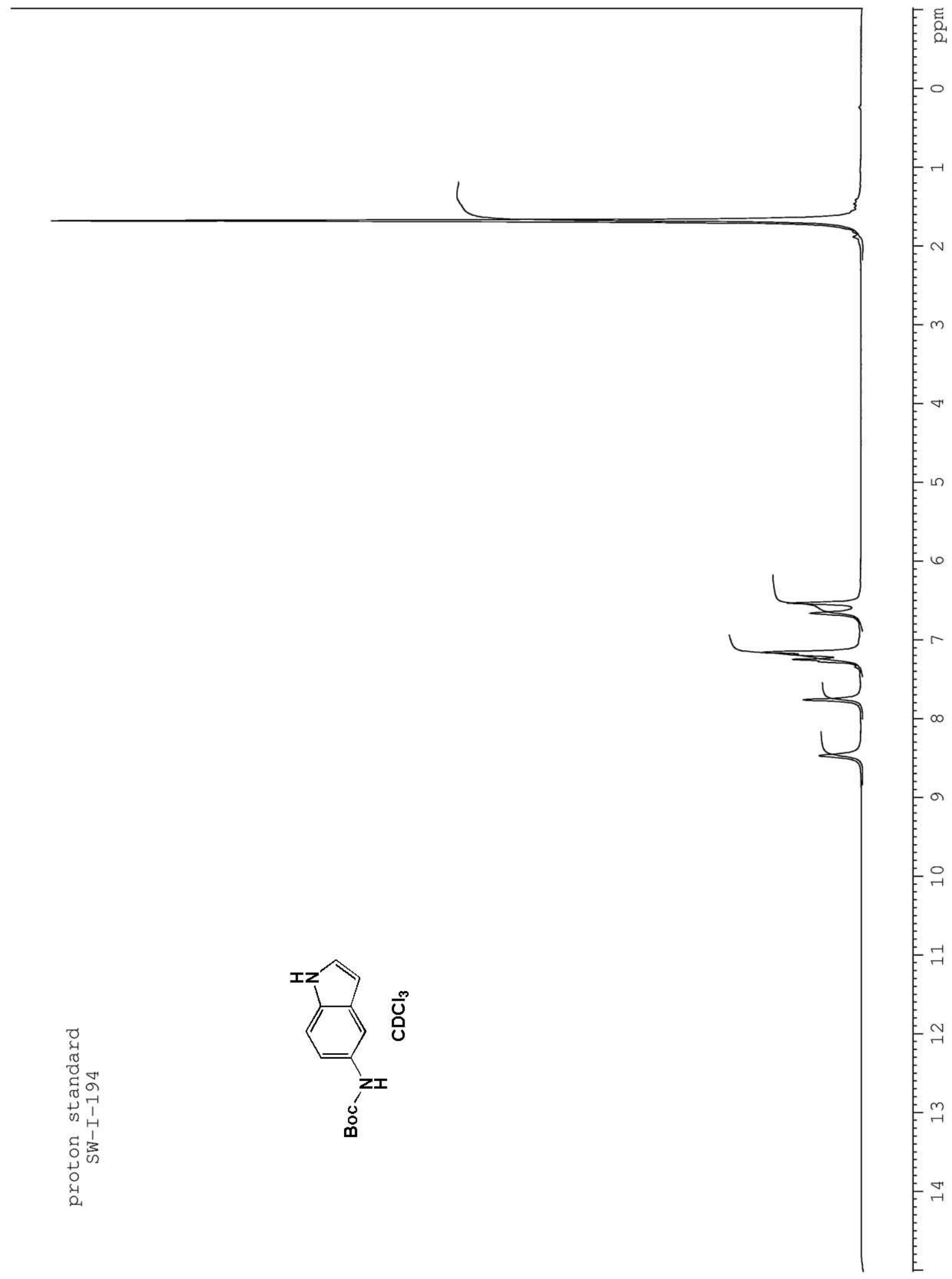




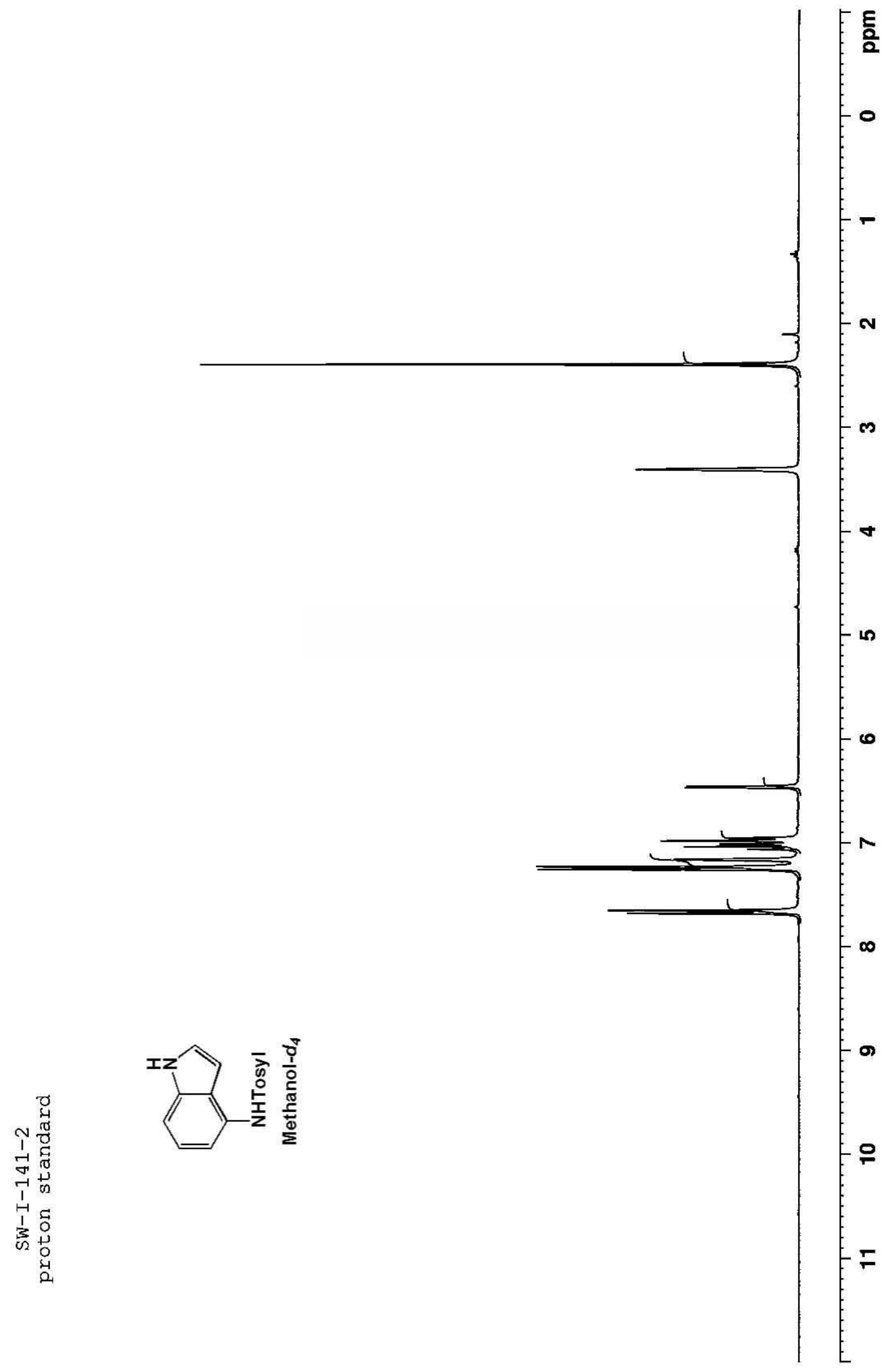




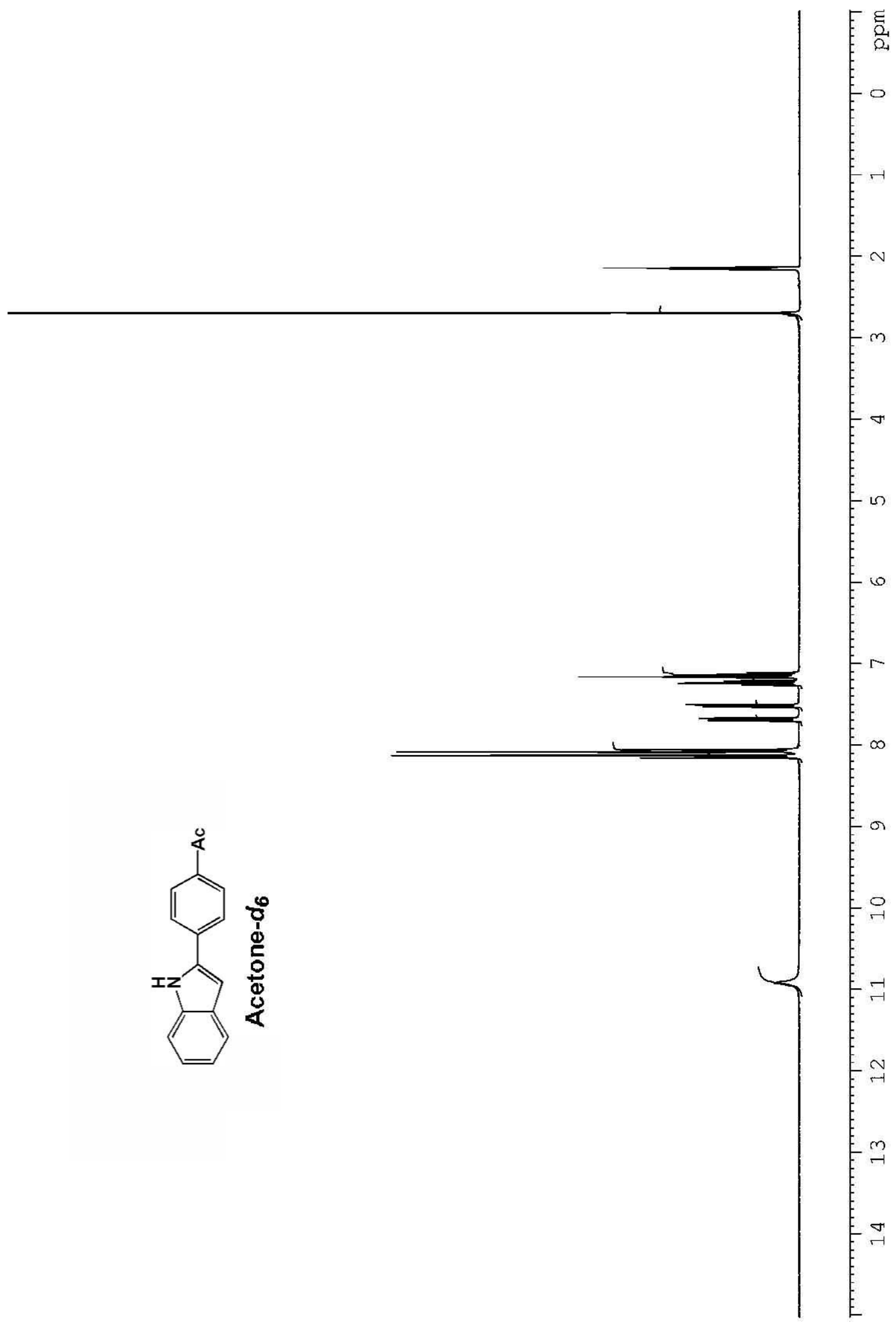




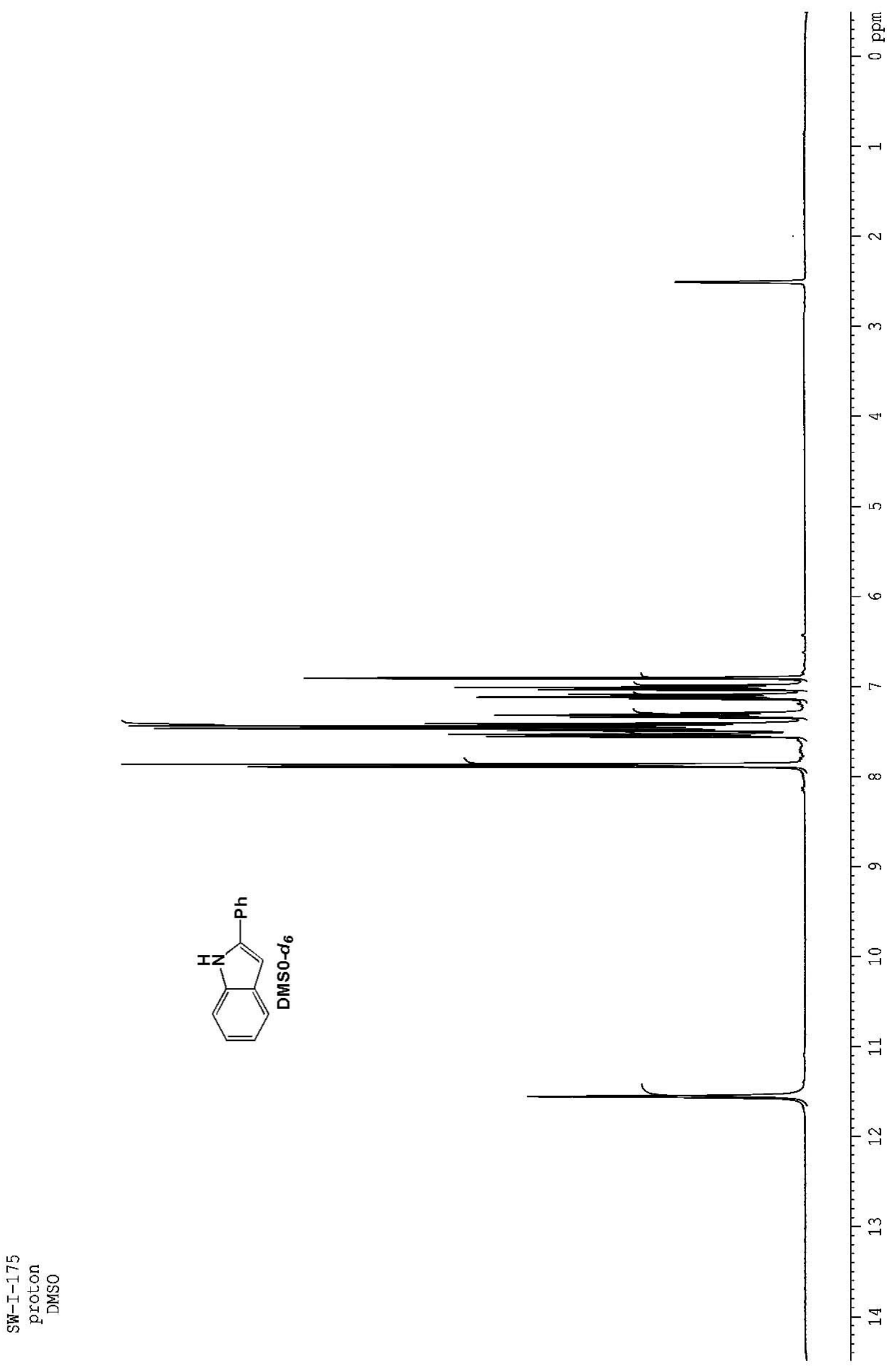




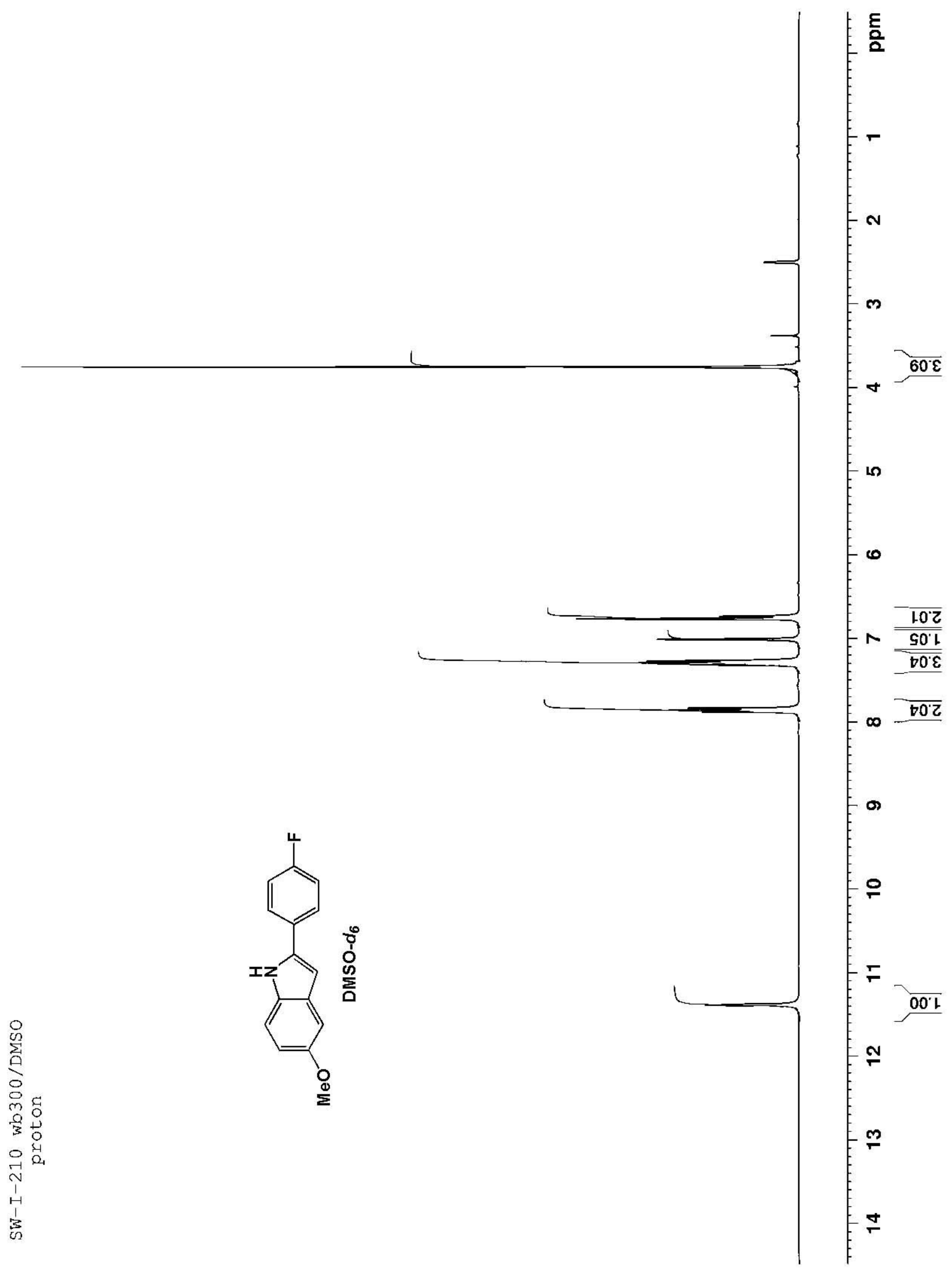




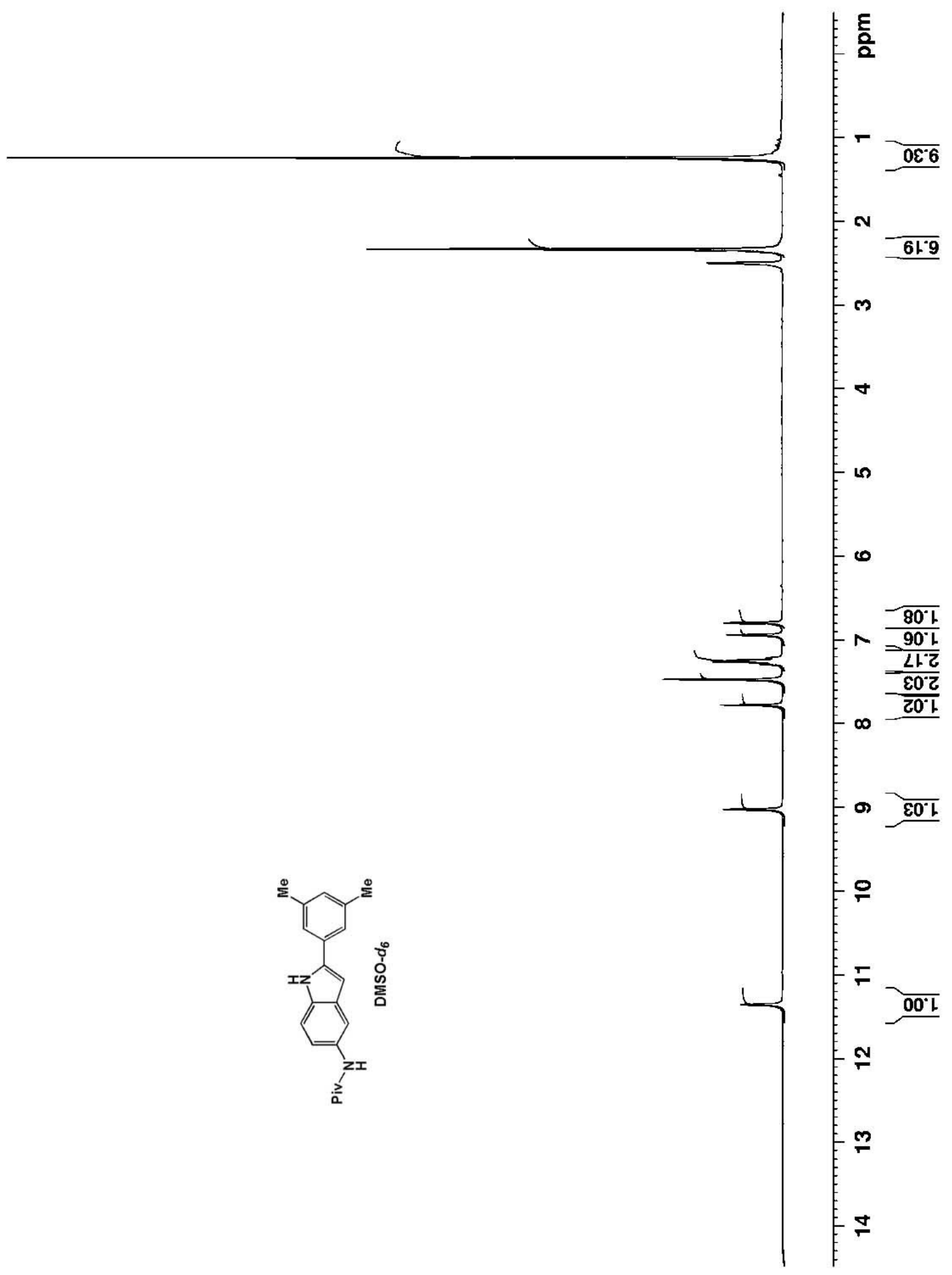




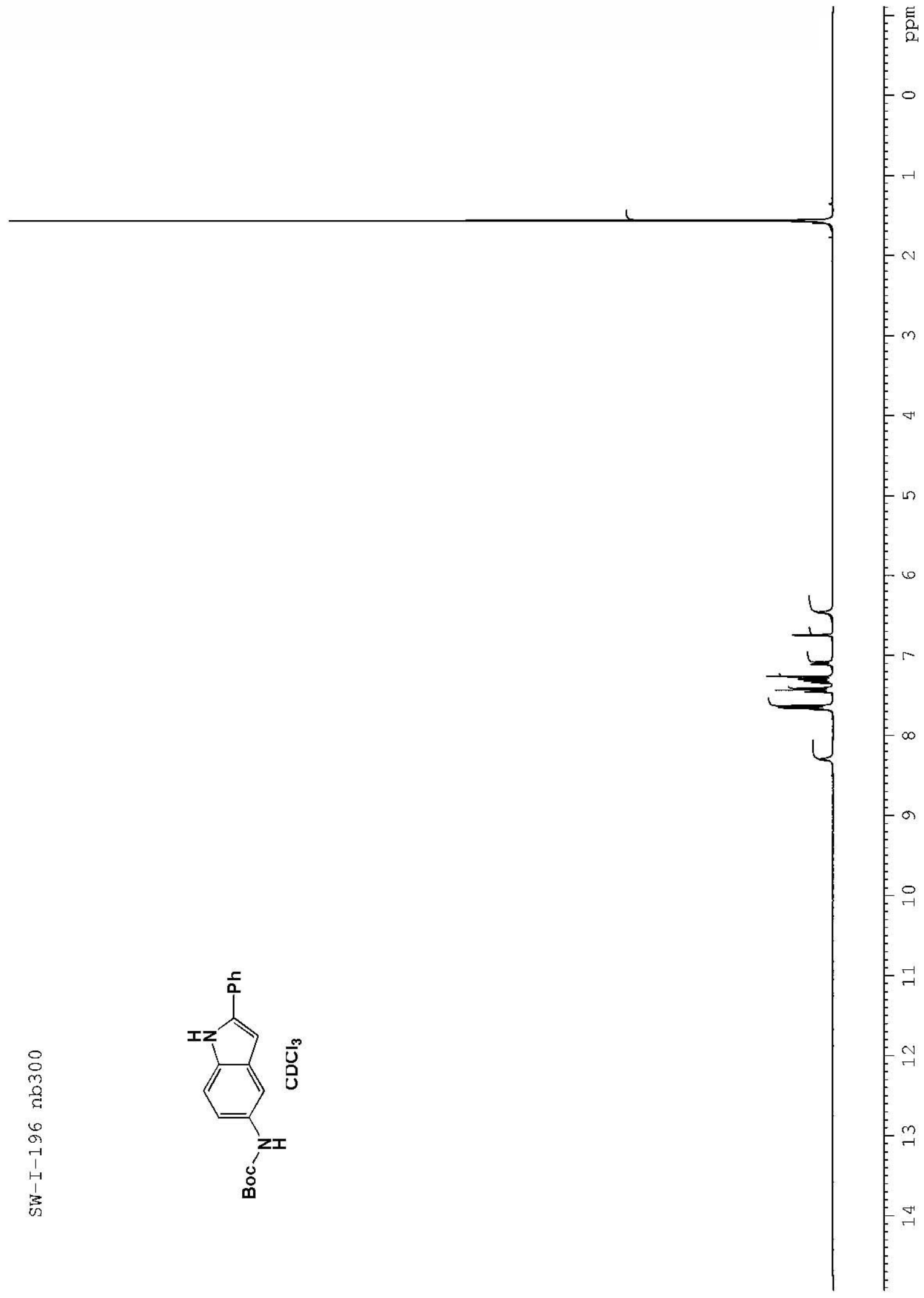




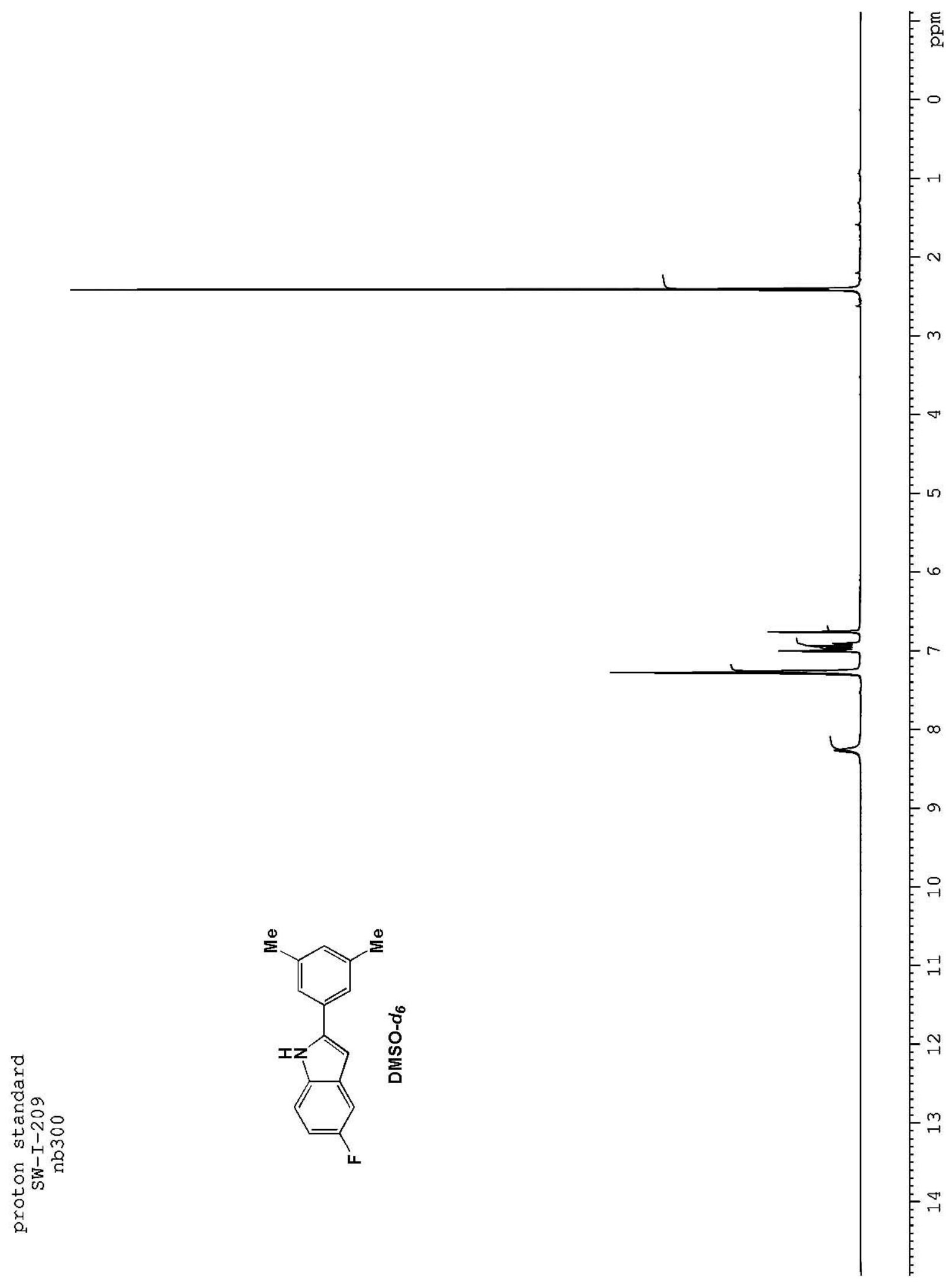




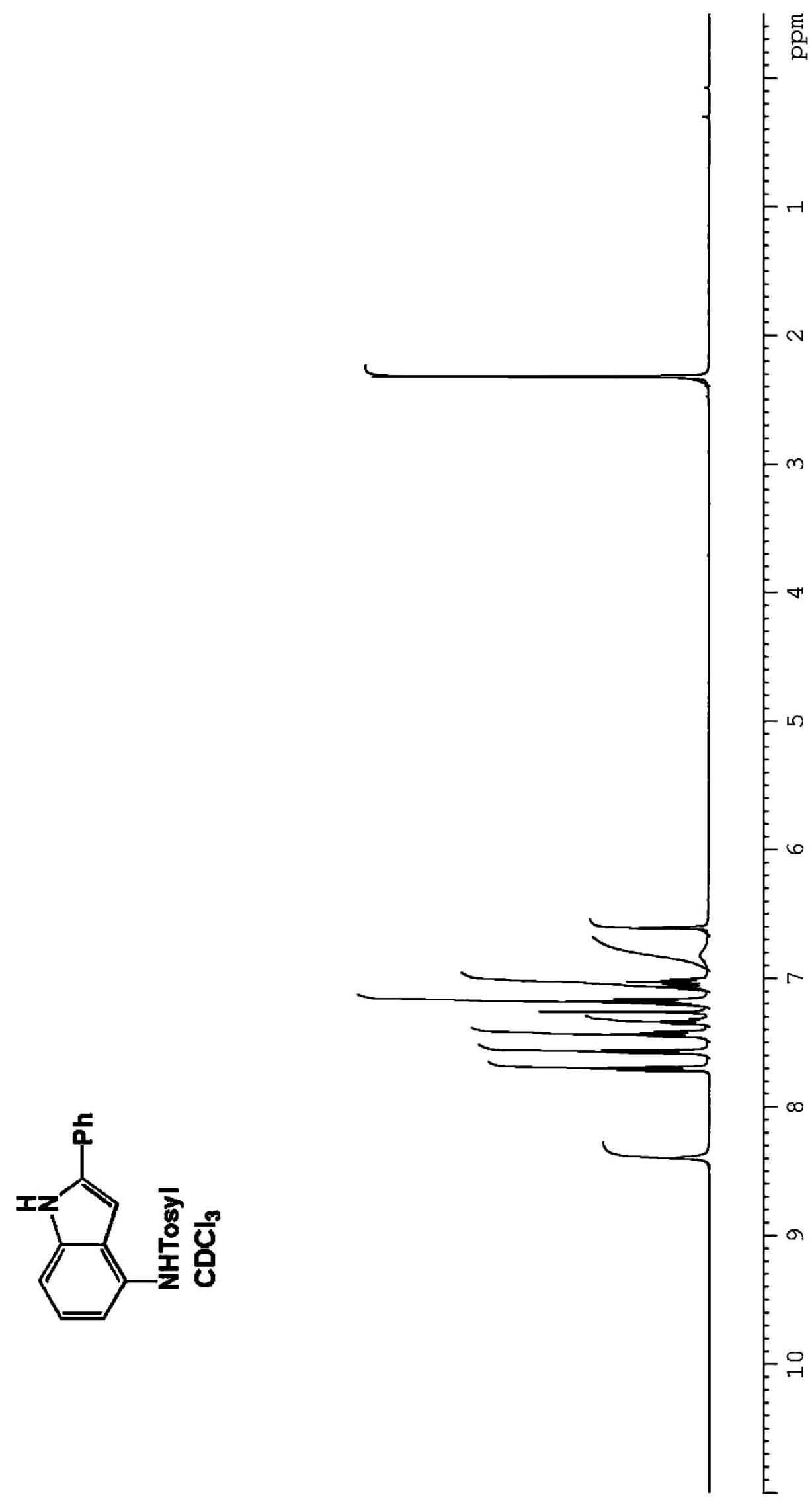




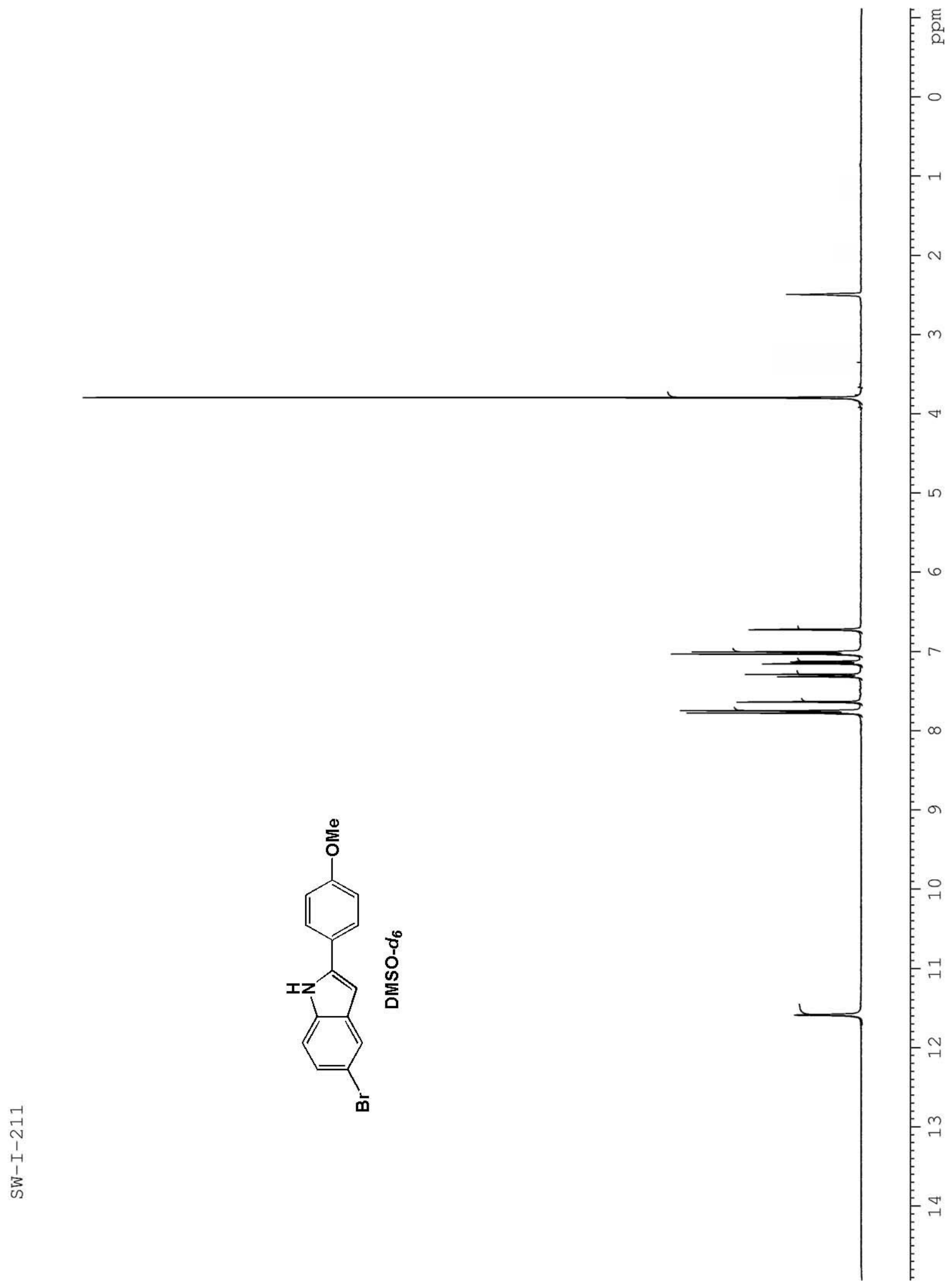




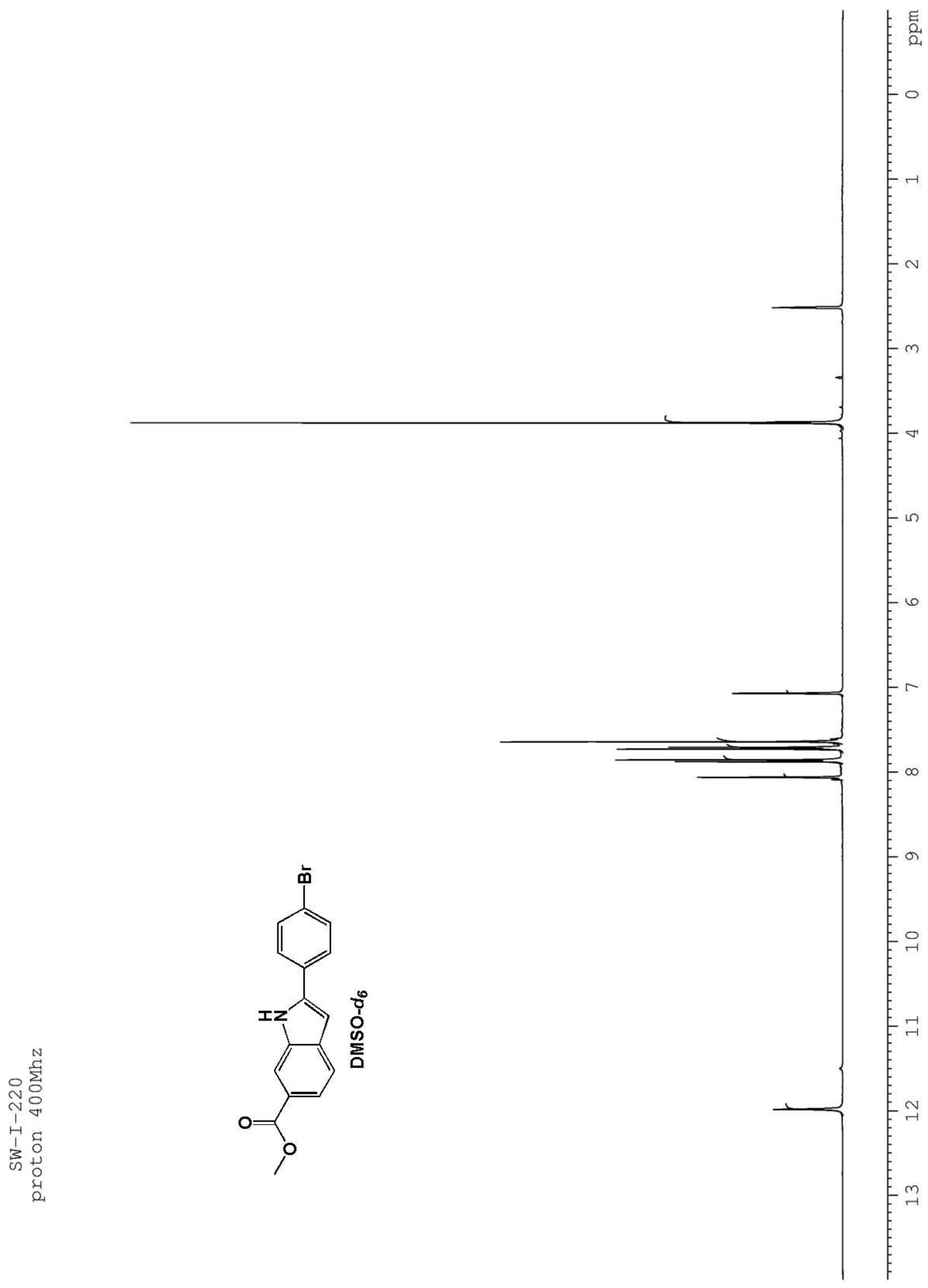




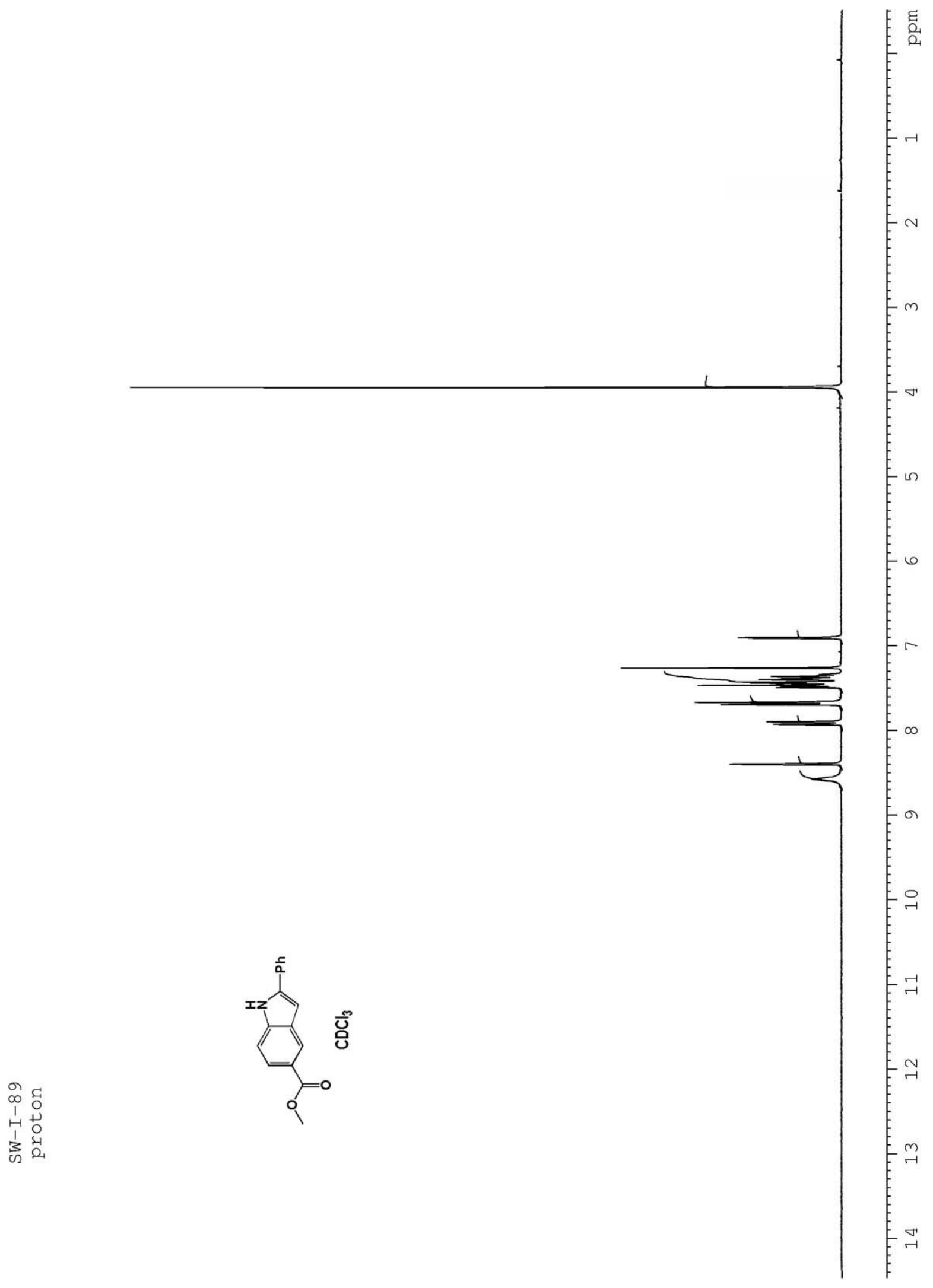




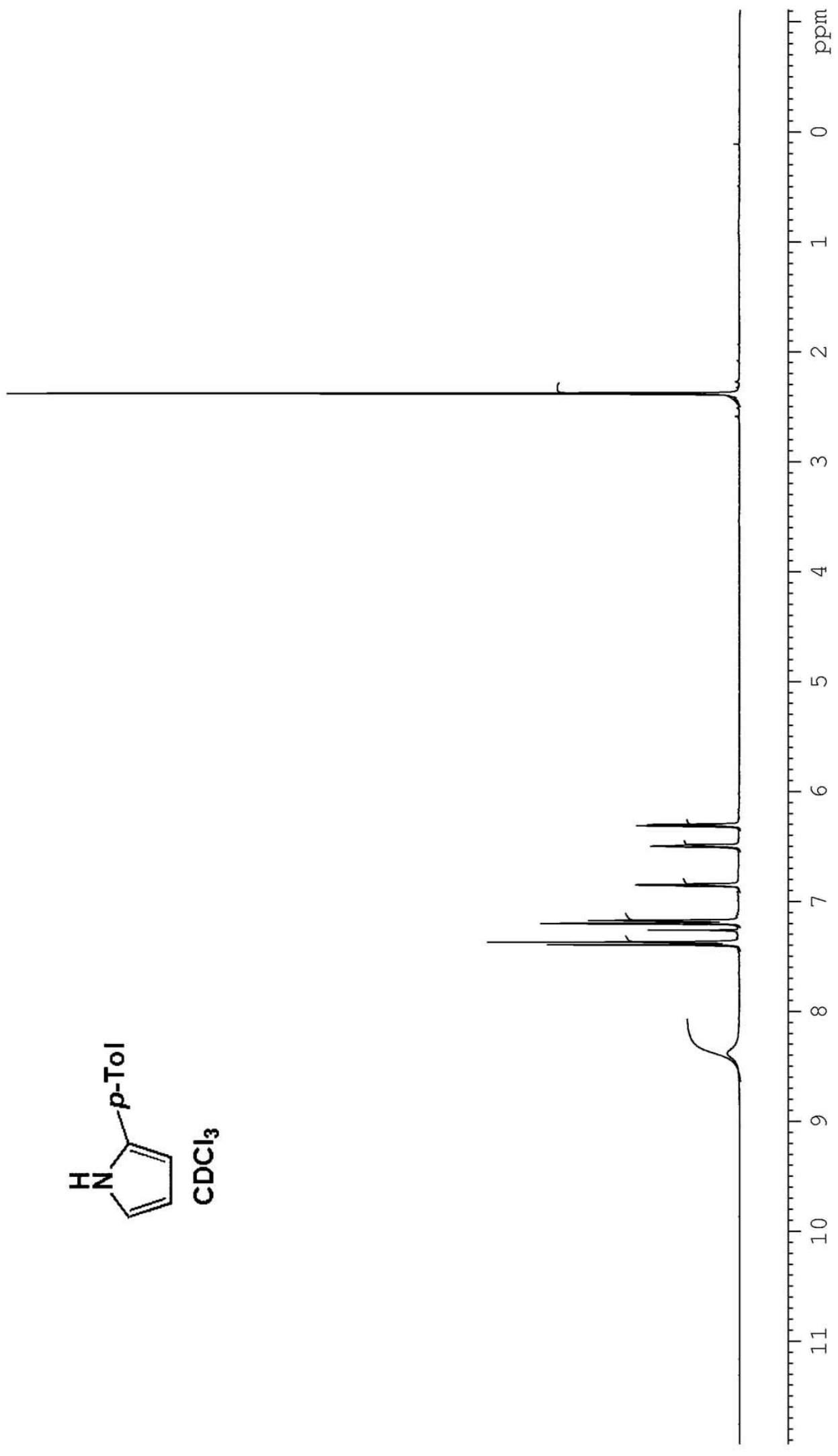




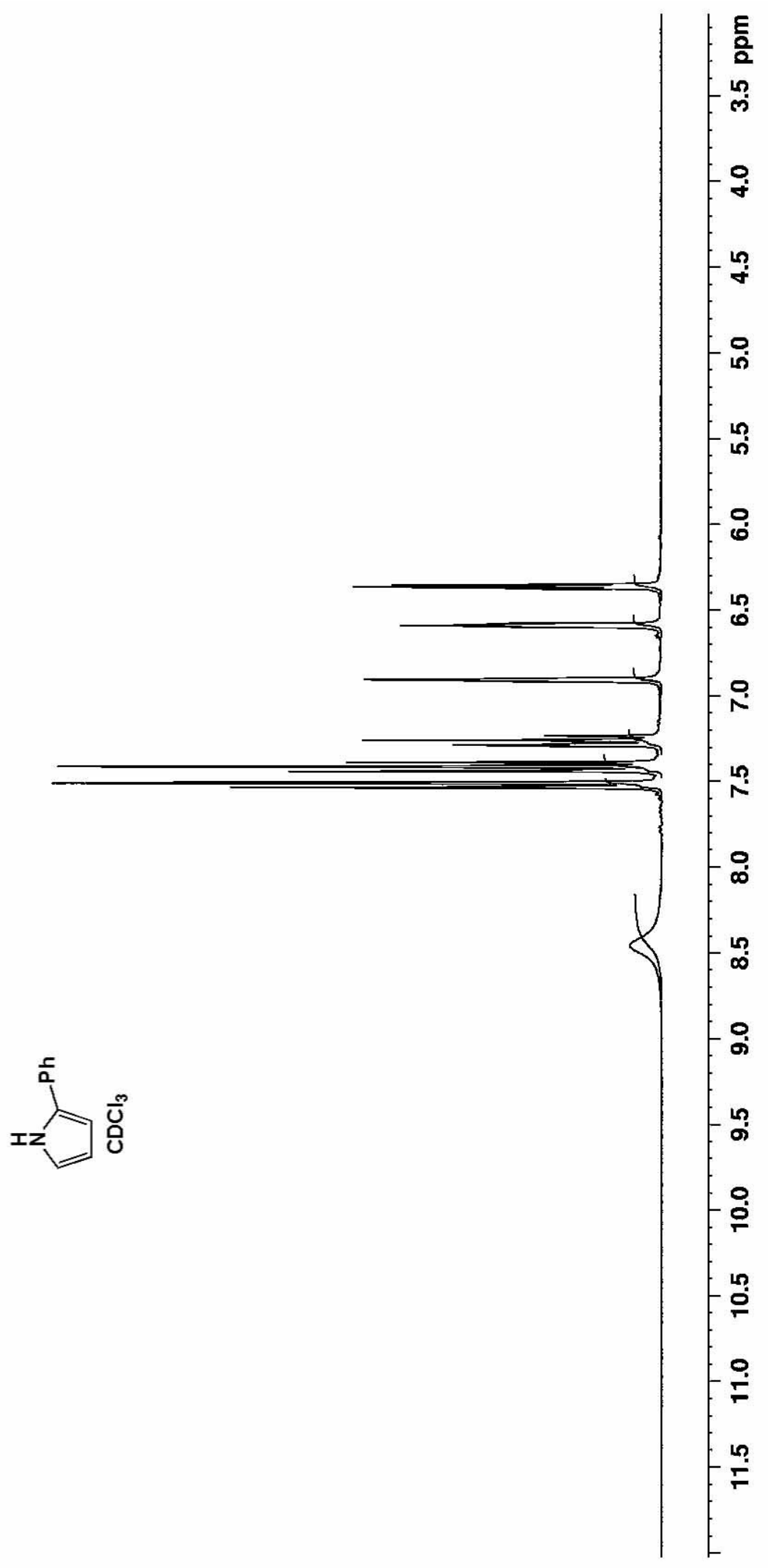




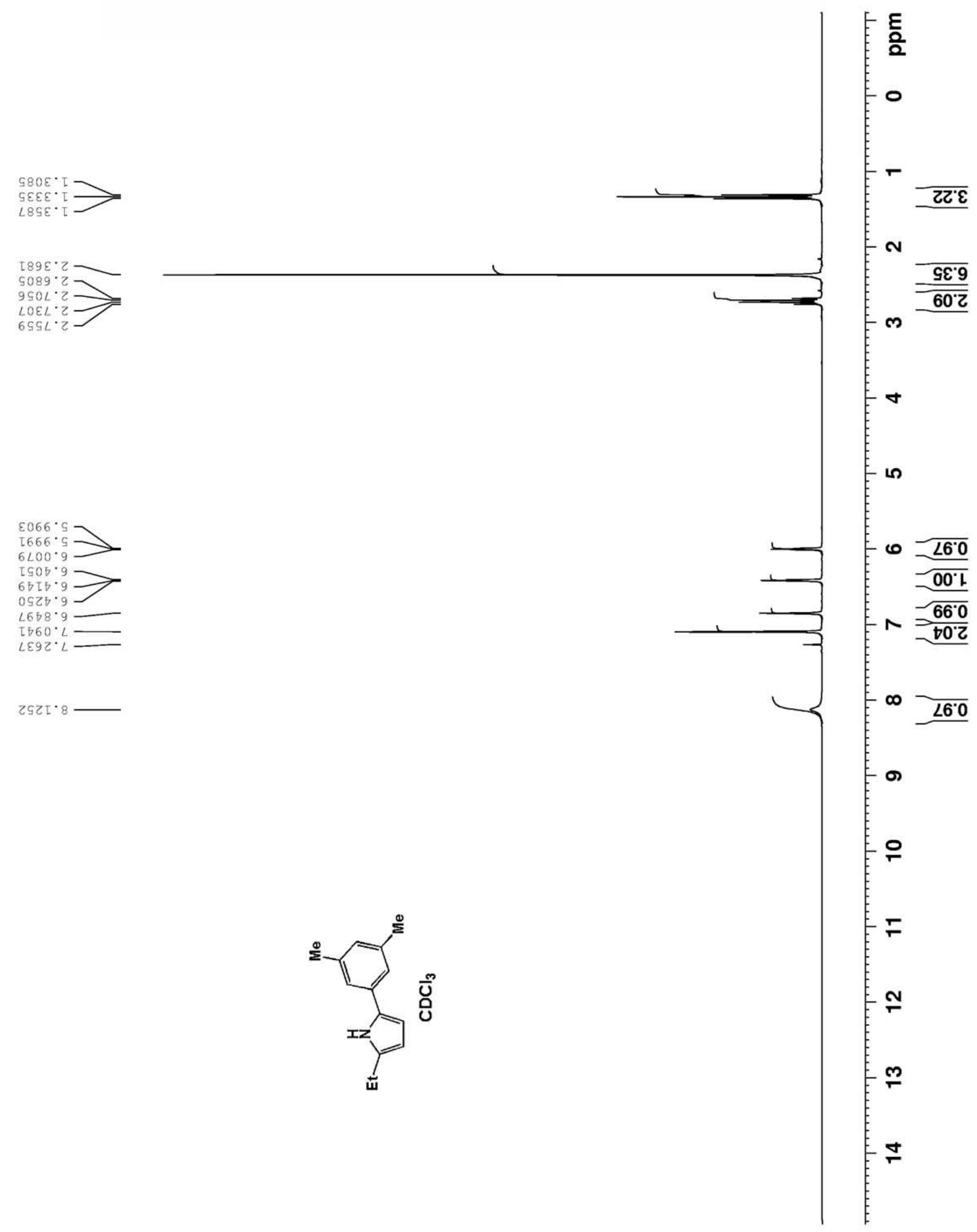




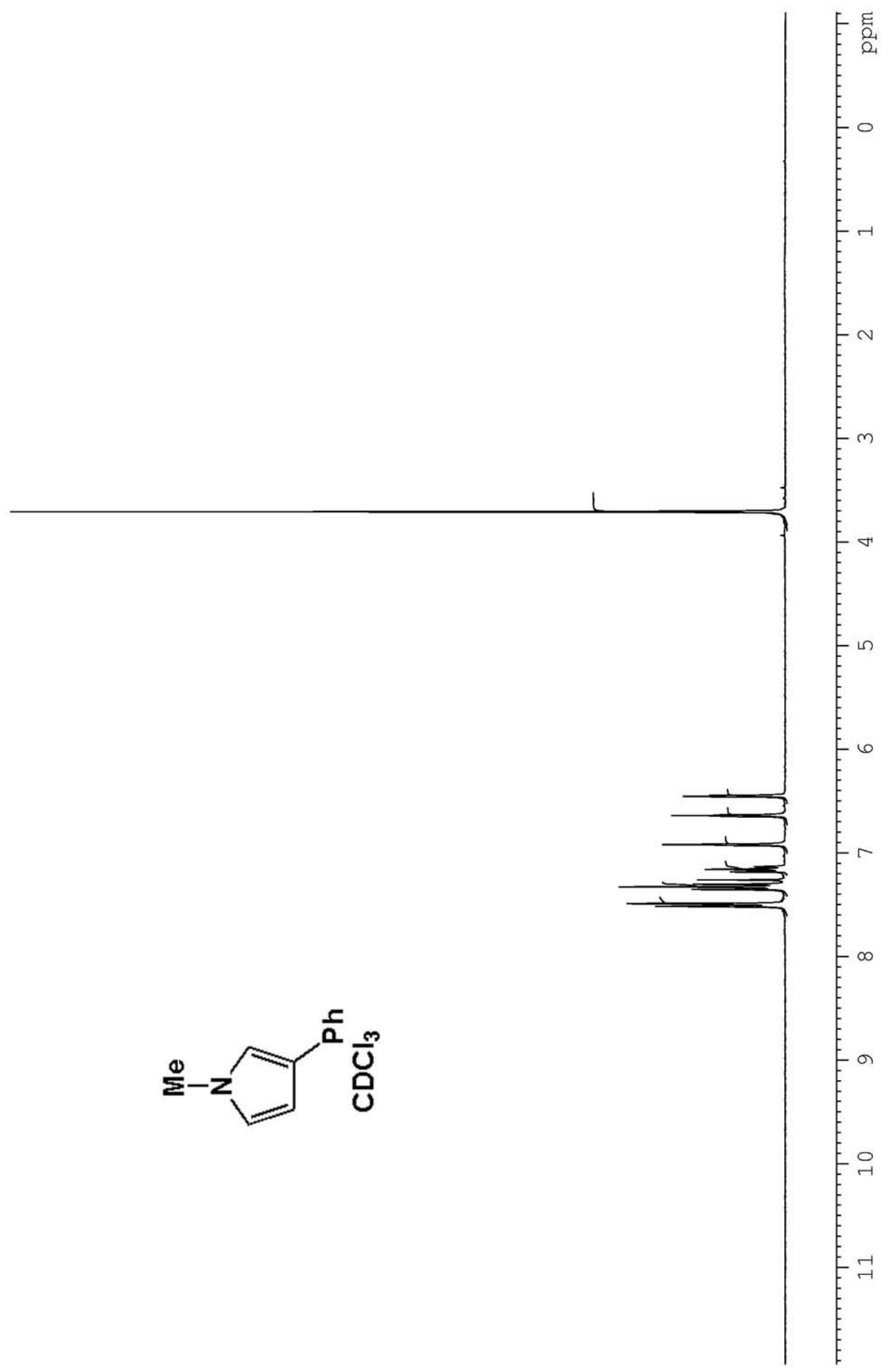




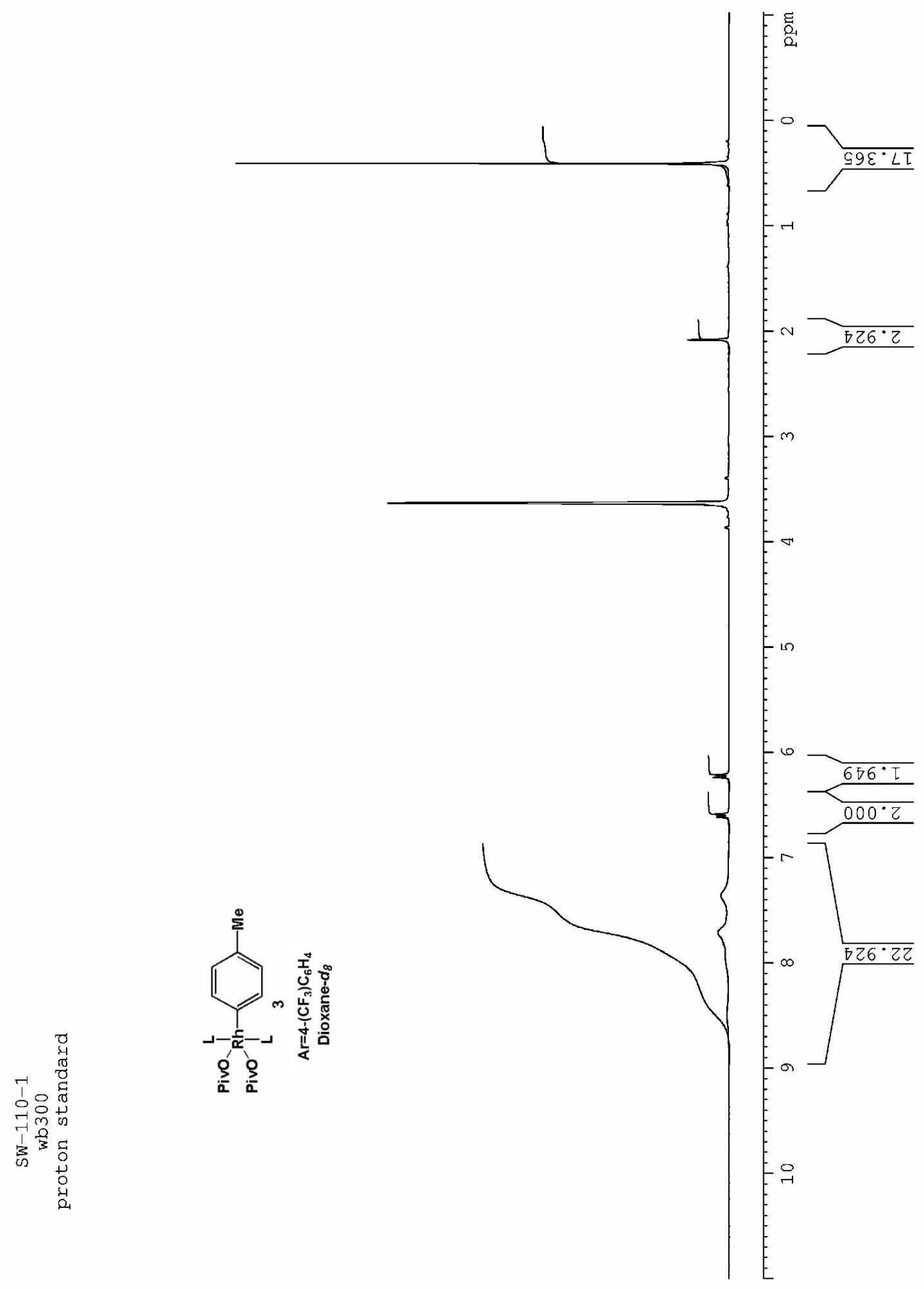




\section{Reference:}

1. Suomalainen, P.; Reinius, H. K.; Riihimaki, H.; Laitinen, R. H.; Jaaskelainen, S.; Haukka, M.; Pursiainen, J. T.; Pakkanen, T. A.; Krause, A. O. I. J. Mol. Cat. A. 2001, 169, 67-78.

2. Van der Ent, A.; Onderdelinden, A. L. Inorg. Synth. 1990, 28, 90-2.

3. Campo, M. A.; Larock, R. C. Org. Lett. 2000, 2, 3675-3677.

4. Bubnov, Y. N.; Zhun, I. V.; Klimkina, E. V.; Ignatenko, A. V.; Starikova, Z. A. Eur. J. Org. Chem. 2000, 3323-3327.

5. Ibaceta-Lizana, J. S. L.; Jackson, A. H.; Prasitpan, N.; Shannon, P. V. R. J. Chem. Soc., Perkin Trans. 2 1987, 1221-6.

6. Sezen, B.; Sames, D. J. Am. Chem. Soc. 2003, 125, 5274-5275.

7. Fagnola, M. C.; Candiani, I.; Visentin, G.; Cabri, W.; Zarini, F.; Mongelli, N.;

Bedeschi, A. Tetrahedron Lett. 1997, 38, 2307-2310.

8. Katritzky, A. R.; Li, J.; Gordeev, M. F. Synthesis 1994, 93-6.

9. Xu, Z.; Lu, X. J. Org. Chem. 1998, 63, 5031-5041.

10. Hickson, C. L.; McNab, H. J. Chem. Soc., Perkin Trans. 1 1988, 339-42.

11. Bonnaud, B.; Bigg, D. C. H. Synthesis 1994, 465-7. 